\title{
Sulphur abundance determinations in star-forming regions - I. Ionization correction factor
}

\author{
O. L. Dors, ${ }^{1 \star}$ E. Pérez-Montero, ${ }^{2 \star}$ G. F. Hägele,,${ }^{3,4}$ M. V. Cardaci ${ }^{3,4}$ and A. C. Krabbe ${ }^{1}$ \\ ${ }^{1}$ Universidade do Vale do Paraíba, Av. Shishima Hifumi, 2911, Cep12244-000, São José dos Campos, SP, Brazil \\ ${ }^{2}$ Instituto de Astrofísica de Andalucía (CSIC), PO Box 3004, E-18080 Granada, Spain \\ ${ }^{3}$ Instituto de Astrofísica de La Plata (CONICET-UNLP), Argentina \\ ${ }^{4}$ Facultad de Ciencias Astronómicas y Geofísicas, Universidad Nacional de La Plata, Paseo del Bosque s/n, 1900 La Plata, Argentina.
}

Accepted 2015 December 22. Received 2015 December 16; in original form 2015 September 21

\begin{abstract}
In this work, we used a grid of photoionization models combined with stellar population synthesis models to derive reliable ionization correction factors (ICFs) for the sulphur in starforming regions. These models cover a large range of nebular parameters and yielding ionic abundances in consonance with those derived through optical and infrared observational data of star-forming regions. From our theoretical ICFs, we suggested an $\alpha$ value of $3.27 \pm 0.01$ in the classical Stasińska formulae. We compared the total sulphur abundance in the gas phase of a large sample of objects by using our theoretical ICF and other approaches. In average, the differences between the determinations via the use of the different ICFs considered are similar to the uncertainties in the $\mathrm{S} / \mathrm{H}$ estimations. Nevertheless, we noted that for some objects it could reach up to about $0.3 \mathrm{dex}$ for the low-metallicity regime. Despite of the large scatter of the points, we found a trend of S/O ratio to decrease with the metallicity, independently of the ICF used to compute the sulphur total abundance.
\end{abstract}

Key words: galaxies: abundances - galaxies: evolution-galaxies: formation-galaxies: general-galaxies: ISM.

\section{INTRODUCTION}

The knowledge of the abundance of heavy elements (e.g. O, S, N, $\mathrm{Ne}$ ) in the gas phase of star-forming regions play a key role in studies of stellar nucleosynthesis, initial mass function (IMF) of stars and chemical evolution of galaxies.

To derive the total abundance of a given element $(\mathrm{X})$ in ionized nebulae, after to estimate the electron temperature and electron density of the gas phase, it is necessary to calculate the abundance of all its ionization stages (see Osterbrock 1989). However, for the majority of the elements present in star-forming regions, only emission lines of some ionization stages can be measured. In these cases, the use of ionization correction factors (ICFs) is necessary to derive the contribution of unobserved ions, as initially defined by Peimbert \& Costero (1969)

$\operatorname{ICF}\left(\mathrm{X}^{+\mathrm{i}}\right)=\frac{\mathrm{X} / \mathrm{H}}{\mathrm{X}^{+\mathrm{i}} / \mathrm{H}^{+}}$,

where $\mathrm{X}^{+\mathrm{i}}$ is the ion whose ionic abundance can be calculated from its observed emission lines.

In particular, for sulphur, in the most of the cases the total abundance is calculated by a direct determination of the abundance of

^E-mail: olidors@univap.br (OLD); epm@iaa.es (EP-M) the ions $\mathrm{S}^{+}$and $\mathrm{S}^{2+}$, through the lines [ $\left.\mathrm{S}_{\mathrm{II}}\right] \lambda \lambda 6716,31$ and $\left[\mathrm{S}_{\mathrm{III}}\right]$ $\lambda \lambda 9069,9532$ respectively, and by using an ICF to correct the unobserved $\mathrm{S}^{3+}$, which produces forbidden lines at $10.51 \mu \mathrm{m}$. In the pioneer work, Stasińska (1978a) proposed an ICF for the sulphur based on both $\mathrm{S}^{+}$and $\mathrm{S}^{2+}$ ions and given by

$\mathrm{ICF}\left(\mathrm{S}^{+}+\mathrm{S}^{2+}\right)=\left[1-\left(1-\frac{\mathrm{O}^{+}}{\mathrm{O}}\right)^{\alpha}\right]^{-1 / \alpha}$

Along decades, the value of $\alpha$ have been largely discussed in the literature. For example, Stasińska (1978a), using the photoionization models of Stasińska (1978b), which assume the non-local thermodynamic equilibrium (NLTE) stellar atmosphere models of Mihalas (1972), suggested $\alpha=3$. French (1981), who used a sample of $\mathrm{H}_{\text {II }}$ regions and planetary nebulae, derived $\alpha=2$. Garnett (1989) combined spectroscopic data of $\mathrm{H}_{\text {II }}$ regions containing the [ $\left.\mathrm{S}_{\mathrm{III}}\right]$ $\lambda \lambda$ 9069, 9532 emission lines (not considered by most of previous works) with photoionization models assuming different stellar atmosphere models in order to estimate an ICF for the sulphur. From this analysis, Garnett (1989) suggested that an intermediary $\alpha$ value between 2 and 3 is correct. Vermeij \& van der Hulst (2002), using the optical and infrared (IR) spectroscopic data of Vermeij et al. (2002), were able to derive directly an ICF for the sulphur and concluded that $\alpha=3$ is correct for $\mathrm{O}^{+} / \mathrm{O}>0.2$, being their results less clear for higher ionization stages (see also Dennefeld \& 
Stasińska 1983; Izotov, Thuan \& Lipovetsky 1994; Thuan, Izotov \& Lipovetsky 1995; Kwitter \& Henry 2001; Kennicutt, Bresolin \& Garnett 2003; Pérez-Montero et al. 2006). Direct estimations for the sulphur ICF, such as the one performed by Vermeij \& van der Hulst (2002), require IR spectroscopic data of $\mathrm{H}$ in regions as well as direct measures of electron temperatures, difficult for objects with low-ionization degrees (Bresolin et al. 2005). Thus, sulphur ICFs have been mainly calculated by using photoionization models, in which no comparisons with observational data are performed.

Other important subject is the relative abundance between sulphur and oxygen, which has a direct impact on studies of stellar nucleosynthesis. These elements arise from the nucleosynthesis in massive stars (Arnett 1978; Woosley \& Weaver 1995); however, there are two fundamental issues ill-defined: (1) the knowledge of the mass range of stars that dominates the production of these elements; (2) if the IMF of stars is universal. For decades, studies based on optical spectroscopic data of star-forming regions have been used to solve these problems but, not conclusive results were obtained. For example, Garnett (1989), who derived sulphur abundances for a sample of 13 extragalactic $\mathrm{H}$ II regions, found a constant S/O abundance over a range of $\mathrm{O} / \mathrm{H}$ (generally used as metallicity tracer), which suggests that either these elements are produced by massive stars within a similar mass range or by stars of different masses but with an universal IMF (Henry \& Worthey 1999). This result is supported by the majority of other works done in this direction (e.g. Kennicutt et al. 2003; Pérez-Montero et al. 2006; Guseva et al. 2011; Berg et al. 2013). However, evidences of S/O ranges with $\mathrm{O} / \mathrm{H}$ were found, for example, by Vílchez et al. (1988) in the galaxy M33 and by Díaz et al. (1991) in M51. Moreover, due to large dispersion in S/O for a fixed value of $\mathrm{O} / \mathrm{H}$ (see e.g. Hägele et al. 2006, 2008, 2012), the idea that S/O does not range with the metallicity is somewhat uncertain (Kehrig et al. 2006; Pérez-Montero et al. 2006).

In this paper, we employ a grid of photoionization models of $\mathrm{H}$ II regions and a large sample of optical and IR spectroscopic data of star-forming regions with the following goals.

(1) To derive ICFs for the sulphur based on a consistent comparison between ionic abundances predicted by photoionization model and calculated from observational data.

(2) To compare the discrepancy in $\mathrm{S} / \mathrm{H}$ abundances computed by using different ICFs.

(3) To investigate the $\mathrm{S} / \mathrm{O}-\mathrm{O} / \mathrm{H}$ relation in star-forming regions considering different ICFs for the sulphur.

This paper is the first (Paper I) of a series of three works, where in the out-coming papers we will present a comparison of $\mathrm{S}^{2+} / \mathrm{H}^{+}$ abundances obtained from optical and IR lines and a comparison between $\mathrm{S} / \mathrm{O}$ and $\mathrm{O} / \mathrm{H}$ abundances with prediction of chemical evolution models. Similar analysis was performed for the neon by Dors et al. (2013). This paper is organized as follows. In Section 2, the observational data used along the paper are presented. In Section 3, we describe the photoionization models used to derive ICFs for the sulphur, while methodology adopted to derive the ionic abundances is given in Section 4. In Section 5, the results containing the ICFs obtained by using photoionization models and from observational emission lines are presented. Discussion and conclusions regarding the outcome are given in Sections 6 and 7, respectively.

\section{OBSERVATIONAL DATA}

We compiled from the literature emission-line intensities of $\mathrm{H}_{\mathrm{II}}$ regions and star-forming galaxies obtained in the optical and IR

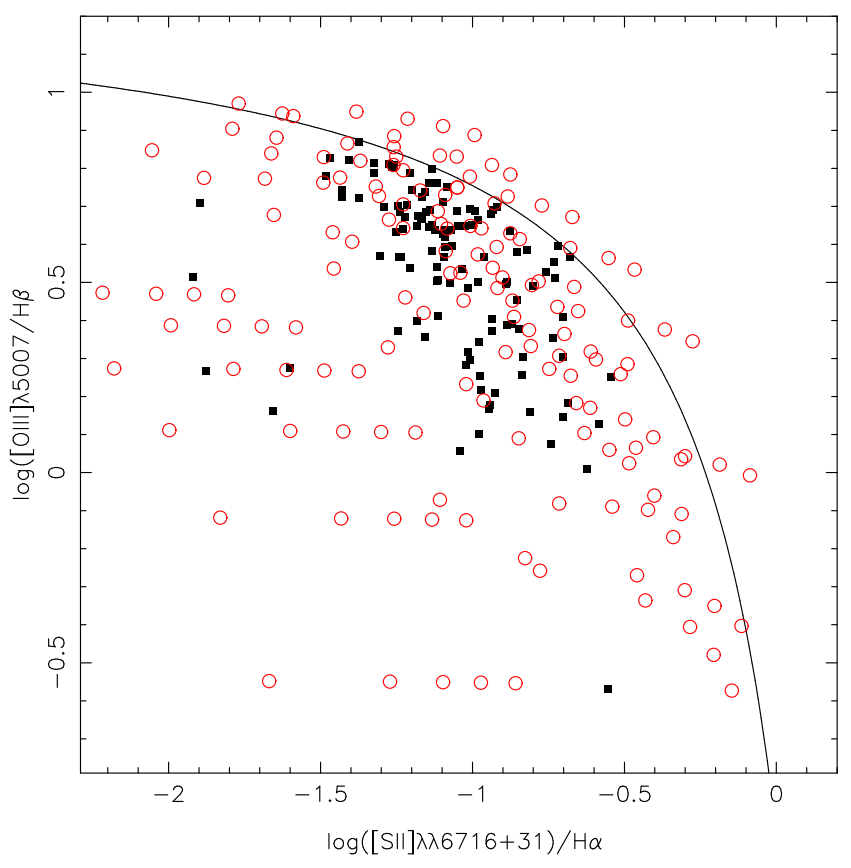

Figure 1. $\log \left[\mathrm{O}_{\mathrm{III}}\right] \lambda 5007 / \mathrm{H} \beta$ versus $\log \left(\left[\mathrm{S}_{\mathrm{II}}\right] \lambda \lambda 6717+31 / \mathrm{H} \alpha\right)$ diagnostic diagram. Solid line, taken from Kewley et al. (2001), separates objects ionized by massive stars from those containing active nuclei and/or shockexcited gas. Black squares represent the objects in our sample. Open circles represent estimations predicted by our models (see Section 3 ).

spectral ranges. These measurements were used to obtain sulphur and oxygen ionic abundances in order to verify if our photoionization models are representative of real $\mathrm{H}_{\text {II }}$ regions, to check if the theoretical ICFs are compatible with the ones derived directly from observations and investigating the $\mathrm{S} / \mathrm{O}-\mathrm{O} / \mathrm{H}$ relation. The selection criterion for the Visible sample was the detection of the intensity lines [O II] $\lambda \lambda 3726+29$ (hereafter refereed as [O II] $\lambda 3727$ ), [O III] $\lambda \lambda 4363,5007,[\mathrm{~S}$ II] $\lambda \lambda 6717,31$ and [S III] $\lambda 9069$. In the cases where the $[\mathrm{S} \mathrm{II}] \lambda 6717,6731$ lines were not resolved, the sum of the intensity of these lines were considered. For some objects (indicated in Table A1) the theoretical relation $I[\mathrm{~S}$ III $] \lambda 9069=I\left[\mathrm{~S}_{\mathrm{III}}\right] \lambda$ 9532/2.5 was used to estimate the emission-line intensity of $\lambda 9069$, since only the sum of these was available.

Since $\mathrm{H}$ II regions and star-forming galaxies are indistinguishable in diagnostic diagrams (e.g. Dors et al. 2013), these objects were considered jointly in our analysis. To eliminate objects with a secondary ionizing source, we use the criterion proposed by Kewley et al. (2001) to distinguish objects ionized by massive stars from those containing an active galactic nucleus and/or gas shock. Hence all objects with

$\log [\mathrm{O}$ III $] \lambda 5007 / \mathrm{H} \beta<\frac{0.72}{\left[\log \left(\left[\mathrm{S}_{\mathrm{II}}\right] \lambda \lambda 6717+31 / \mathrm{H} \alpha\right)\right]-0.32}$

were selected. In Fig. 1, the objects in our sample and a curve representing the criterion above are shown.

In the appendix, Table A1 lists the object identification, optical emission-line intensities (relative to $\mathrm{H} \beta=100$ ) and bibliographic references of the sample. We obtained optical data of 118 objects. All emission-line intensities were reddening corrected by the authors of the original works from which we have taken the data. Dors et al. (2013) showed that effects of using heterogeneous data sample, such as the one used in this paper, do not yield any bias on the 
results of abundance estimations in the gas phase of star-forming regions.

We also considered emission-line intensities of $143 \mathrm{H}$ II galaxies of a sample of 310 galaxies considered by Izotov et al. (2006a) and selected from the Sloan Digital Sky Survey (SDSS; York et al. 2000) Data Release 3. We applied a similar selection criterion above but with small changes due to the shorter wavelength spectral coverage of the Sloan data (when [O II] 33727 is observed [S III] $] 2069$ is not, and vice versa, depending on the object redshift). Hence, we selected the objects that present the [S III] $\lambda 9069$ emission lines and [O II] $\lambda 7325$ instead of [O II] $\lambda 3727$. These objects are also represented in Fig. 1 but are not listed in Table A1.

Concerning the IR sample, the selection criterion was the presence of the flux measurements of the emission lines $\mathrm{H}_{\mathrm{I}} 4.05 \mu \mathrm{m}$, [S IV] $10.51 \mu \mathrm{m}$ and [S III] $18.71 \mu \mathrm{m}$. We compiled IR data of 103 objects classified as being $\mathrm{H}$ II regions and nuclei of galaxies containing star formation regions. Only nine objects have both optical and IR data. In the appendix, Table A2, object identification, fluxes of the emission lines considered and bibliographic references of the sample are listed. In some cases, indicated in Table A2, the $\mathrm{H}_{\mathrm{I}} 4.05 \mu \mathrm{m}$ emission-line fluxes were computed from Н I $12.37 \mu \mathrm{m}$ or $\mathrm{H}_{\text {I }} 2.63 \mu \mathrm{m}$ fluxes, assuming the theoretical ratios Н $4.051 \mu \mathrm{m} / \mathrm{H}_{\text {I }} 12.37 \mu \mathrm{m}=8.2$ and $\mathrm{H}_{\text {I }} 4.051 \mu \mathrm{m} / \mathrm{H}_{\text {I }} 2.63 \mu \mathrm{m}=$ 1.74 taken from Storey \& Hummer (1995) for $N_{\mathrm{e}}=100 \mathrm{~cm}^{-3}$ and $T_{\mathrm{e}}=10000 \mathrm{~K}$.

For the objects with emission-line measurements at different spatial positions, indicated in the Table A2, the adopted fluxes were the sum (integrated) of the individual ones. The purpose of this procedure is to avoid taking exclusive emission lines from outer parts of $\mathrm{H}$ II regions into account, which the diffuse gas emission (e.g. Walterbos 1998; Helmbold et al. 2005) component can be important but it is not considered in our photoionization models.

The aperture sizes in which the optical and IR data were taken for a same object can be different from each other, yielding uncertainties in our results. In fact, Kewley, Jansen \& Geller (2005) presented a detailed analysis of the effect of considering different aperture on determinations of physical parameters of galaxies. They have found that systematic and random errors from aperture effects can arise if fibres capture less than 20 per cent of the galaxy light. Most of the star-forming regions in our sample can be treated as point sources, and almost all the object extensions are observed. Therefore, this effect seems to be negligible for our sample of objects.

\section{PHOTOIONIZATION MODELS}

We built a grid of photoionization models using the CLOUDY code version 13.03 (Ferland et al. 2013) to estimate an ICF for the sulphur. These models are similar to the ones presented by Dors et al. (2011) and in what follows the input parameters are briefly discussed.

(i) Spectral energy distribution - the synthetic spectra of stellar clusters with $1 \mathrm{Myr}$, built with the STARBURST99 (Leitherer et al. 1999) assuming the WM-basic stellar atmosphere models by Pauldrach, Hoffmann \& Lennon (2001), and the 1994 Geneva tracks with standard mass loss with metallicities $Z=1.0,0.4,0.2,0.05 \mathrm{Z}_{\odot}$, were considered.

(ii) Ionization parameter - the ionization parameter $U$ is defined as $U=Q_{\text {ion }} / 4 \pi R_{\text {in }}^{2} n c$, where $Q_{\text {ion }}$ is the number of hydrogen ionizing photons emitted per second by the ionizing source, $R_{\text {in }}$ is the distance from the ionization source to the inner surface of the ionized gas cloud (in $\mathrm{cm}$ ), $n$ is the particle density (in $\mathrm{cm}^{-3}$ ), and $c$ is the speed of light. We assumed $R_{\text {in }}=4 \mathrm{pc}$, a typical size of a stellar cluster and also used by Stasińska \& Izotov (2003) to model a large sample of data of star-forming galaxies. The value $n=200 \mathrm{~cm}^{-3}$ was assumed in the models, a typical value of $\mathrm{H}$ II regions located in discs of isolated galaxies (e.g. Krabbe et al. 2014).

We considered the $\log Q_{\text {ion }}$ ranging from 48 to 54 dex, with a step of 1.0 dex. From the computed sequence of models for the hypothetical nebulae, we found $\log U$ ranging from $\sim-1.5$ to $\sim-4.0$, typical values of $\mathrm{H}_{\text {II }}$ regions (e.g. Bresolin, Kennicutt \& Garnett 1999; Dors et al. 2013; Freitas-Lemes et al. 2014; Rosa et al. 2014; Pérez-Montero 2014; Sánchez et al. 2015).

(iii) Metallicity - the metallicity of the gas phase, $Z$, was linearly scaled to the solar metal composition (Allende Prieto, Lambert \& Asplund 2001) and the values $Z=1.0,0.6,0.4,0.2,0.05 \mathrm{Z}_{\odot}$ were considered. In order to build realistic models, the metallicity of the nebula was matched with the closest available metallicity of the stellar atmosphere (see Dors et al. 2011 for a discussion about this methodology). For the nitrogen, we computed its abundance from the relation between $\mathrm{N} / \mathrm{O}$ and $\mathrm{O} / \mathrm{H}$ given by Vila-Costas \& Edmunds (1993). Although the relation between $\mathrm{N}$ and O presents a high dispersion (e.g. Pérez-Montero \& Contini 2009) this does not affect the results of this study, since we do not use nitrogen emission lines. Since the relation between S/O and metallicity is uncertain (Kehrig et al. 2006; Pérez-Montero et al. 2006), five grids of models were built with the following values of $\log (\mathrm{S} / \mathrm{O}):-1.31$, -1.42 (solar value), $-1.55-1.72$, and -2.12 .

The presence of internal dust was considered and the grain abundances of van Hoof et al. (2001) were linearly scaled with the oxygen abundance. The abundances of the refractory elements $\mathrm{Mg}, \mathrm{Al}, \mathrm{Ca}$, $\mathrm{Fe}, \mathrm{Ni}$ and $\mathrm{Na}$ were depleted by a factor of 10 , and $\mathrm{Si}$ by a factor of 2 , relative to the adopted abundances of the gas phase in each model. The resulting geometry was spherical in all models. In total, 175 photoionization models were built. In Fig. 1, intensities of the line ratios $\log \left(\left[\mathrm{O}_{\mathrm{III}}\right] \lambda 5007 / \mathrm{H} \beta\right)$ and $\log ([\mathrm{S}$ II] $] \lambda 26717+31 / \mathrm{H} \alpha)$ predicted by the models are also plotted, where it can be seen that the models cover very well the region occupied by the observations.

\section{DETERMINATION OF IONIC ABUNDANCES}

Using the observational data in Table A1, the ionic abundances of $\mathrm{O}^{+}, \mathrm{O}^{2+}, \mathrm{S}^{+}$and $\mathrm{S}^{2+}$ were computed using direct estimations of the electron temperatures (following Dors et al. 2013, this method will be called the Visible-lines method). We also used the observational data in Table A2 to calculate the $\mathrm{S}^{2+}$ and $\mathrm{S}^{3+}$ ionic abundances through IR emission lines (this method will be called the IR-lines method). In what follows, a description of each method is given.

\subsection{Visible-lines method}

For the objects listed in Table A1, the electron temperature values and oxygen and sulphur ionic abundances were derived from the expressions obtained by Pérez-Montero (2014) and by using the same atomic parameters used in the version 13.03 of the CLOUDY code and listed in Table 1. These parameters were included in the PYNEB code (Luridiana, Morisset \& Shaw 2015) to derive the oxygen and sulphur abundances as a function of emission-line ratios and electron temperature. These equations are valid for the electron temperature range 8000-25 $000 \mathrm{~K}$ and they are presented in what follows.

For the objects listed in Table A1, we calculated the electron temperature $\left(T_{\mathrm{e}}\right)$ from the observed line-intensity ratio $R_{\mathrm{O} 3}=(1.33 \times$ $\left.I\left[\mathrm{O}_{\mathrm{III}}\right] \lambda 5007\right) / I\left[\mathrm{O}_{\mathrm{III}}\right] \lambda 4363$ for the high-ionization zone (refereed 
Table 1. Sources of the atomic data of sulphur and oxygen ions.

\begin{tabular}{lcc}
\hline \multicolumn{2}{c}{ References } \\
Ion & Transition probabilities & Collisional strengths \\
\hline $\mathrm{S}^{+}$ & Podobedova, Kelleher \& Wiese (2009) & Tayal \& Zatsarinny (2010) \\
$\mathrm{S}^{2+}$ & Podobedova et al. (2009) & Tayal \& Gupta (1999) \\
$\mathrm{S}^{3+}$ & Johnson, Kingston \& Dufton (1986) & Tayal (2002) \\
$\mathrm{O}^{+}$ & Zeippen (1982) & Pradhan et al. (2006) \\
$\mathrm{O}^{2+}$ & Storey \& Zeippen (2000) & Aggarwal \& Keenan (1999) \\
\hline
\end{tabular}

as $t_{3}$ ) using the fitted function:

$t_{3}=0.7840-0.0001357 \times R_{\mathrm{O} 3}+\frac{48.44}{R_{\mathrm{O} 3}}$,

with $t$ in units of $10^{4} \mathrm{~K}$.

Adopting the same methodology of Pérez-Montero (2014), the electron density $\left(N_{\mathrm{e}}\right)$ was computed from the ratio $R_{S 2}=[\mathrm{S}$ II] $\lambda 6716 / \lambda 6731$ and using the following expression proposed by Hägele et al. (2008)

$N_{\mathrm{e}}=10^{3} \times \frac{R_{S 2} \times a_{0}(t)+a_{1}(t)}{R_{S 2} \times b_{0}(t)+b_{1}(t)}$,

with $N_{\mathrm{e}}$ in units of $\mathrm{cm}^{-3}$ and $t$ in units of $10^{4} \mathrm{~K}$.

Using the appropriate fittings and PYNEB with collision strengths listed in Table 1, the coefficients of equation (5) can be written in the form

$a_{0}(t)=16.054-7.79 / t-11.32 \times t_{2}$,

$a_{1}(t)=-22.66+11.08 / t+16.02 \times t_{2}$,

$b_{0}(t)=-21.61+11.89 / t+14.59 \times t_{2}$,

$b_{1}(t)=9.17-5.09 / t-6.18 \times t_{2}$,

being $t_{2}$ defined by

$t_{2}=\frac{1.397}{0.385+t_{3}^{-1}}$.

For the cases where $R_{S 2}$ is unresolved, a value of $N_{\mathrm{e}}=200 \mathrm{~cm}^{-3}$ was assumed.

The $\mathrm{O}^{2+}$ and $\mathrm{O}^{+}$abundances were computed following the relations:

$$
\begin{aligned}
12+\log \left(\frac{\mathrm{O}^{2+}}{\mathrm{H}^{+}}\right)= & \log \left[\frac{I(5007)}{I(\mathrm{H} \beta)}\right]+6.3106 \\
& +\frac{1.2491}{t_{3}}-0.5816 \times \log t_{3}
\end{aligned}
$$

and

$$
\begin{aligned}
12+\log \left(\frac{\mathrm{O}^{+}}{\mathrm{H}^{+}}\right)= & \log \left[\frac{I(3727)}{I(\mathrm{H} \beta)}\right]+5.887 \\
& +\frac{1.641}{t_{2}}-0.543 \times \log t_{2}+10^{-3.94} n_{\mathrm{e}},
\end{aligned}
$$

where $n_{\mathrm{e}}=N_{\mathrm{e}} /\left(10^{4} \mathrm{~cm}^{-3}\right)$.

Concerning the SDSS data taken from Izotov et al. (2006a, not listed in Table A1), for the objects with redshift $z>0.02$ in which the $\left[\mathrm{S}_{\text {III }}\right] \lambda 9069$ was measured, the $\left[\mathrm{O}_{\text {II }}\right] \lambda 3727$ is out of the spectral range. Therefore, for this data set, the $\mathrm{O}^{+}$abundance was computed using the fluxes of the $\left[\mathrm{O}_{\mathrm{II}}\right] \lambda \lambda 7320,7330$ emission lines and the expression also derived using the PYNEB code (Luridiana et al.
2015):

$$
\begin{aligned}
12+\log \left(\frac{\mathrm{O}^{+}}{\mathrm{H}^{+}}\right)= & \log \left[\frac{I(7320+7330)}{I(\mathrm{H} \beta)}\right]+7.21 \\
& +\frac{2.511}{t_{2}}-0.422 \times \log t_{2} \\
& +10^{-3.40} n_{\mathrm{e}}\left(1-10^{-3.44} \times n_{\mathrm{e}}\right) .
\end{aligned}
$$

For the sulphur ionic abundances, the equations used are

$$
\begin{aligned}
12+\log \left(\frac{\mathrm{S}^{+}}{\mathrm{H}^{+}}\right)= & \log \left[\frac{I(6717+6731)}{I(\mathrm{H} \beta)}\right]+5.423 \\
& +\frac{0.941}{t_{2}}-0.37 \log t_{2}
\end{aligned}
$$

and

$$
\begin{aligned}
12+\log \left(\frac{\mathrm{S}^{2+}}{\mathrm{H}^{+}}\right)= & \log \left[\frac{I(9069)}{I(\mathrm{H} \beta)}\right]+6.527 \\
& +\frac{0.661}{t_{S 3}}-0.527 \log t_{S 3} .
\end{aligned}
$$

To derive the $t_{S 3}$ temperature for the gas region where the $S^{2+}$ is located, we used the relation (see Pérez-Montero \& Díaz 2005)

$t_{S 3}=1.05 \times t_{3}-0.08$.

The electron temperature $\left(t_{3}\right)$, electron density and ionic abundances calculated from the preceding equations and using the optical data (Table A1) are listed in Table A3 in the appendix. Typical errors of emission-line intensities are about 10-20 per cent and of electron temperature determinations $\sim 500 \mathrm{~K}$, which yield an uncertainty in ionic abundances of about 0.15 dex (see Vermeij \& van der Hulst 2002; Kennicutt et al. 2003; Hägele et al. 2008). Hereafter, we will assume that the abundances based on Visible-lines method have an uncertainty of 0.15 dex.

\subsection{IR-lines method}

In order to derive more precise ionic sulphur abundances, we have taken into account the temperature dependence on the emission coefficients to derive $\mathrm{S}^{2+}$ and $\mathrm{S}^{3+}$ abundances from IR lines. We computed the $\mathrm{S}^{2+}$ and $\mathrm{S}^{3+}$ ionic fractions from [S III] $18.71 \mu \mathrm{m}$ and [S IV] $10.51 \mu \mathrm{m}$ emission lines, respectively, and considering the line $\mathrm{H}_{\mathrm{I}} 4.05 \mu \mathrm{m}$ presented in Table A2. We used the code PYNEB (Luridiana et al. 2015) and the atomic parameters presented in Table 1 to derive the equations

$$
\begin{aligned}
12+\log \left(\frac{\mathrm{S}^{2+}}{\mathrm{H}^{+}}\right)= & \log \left(\frac{I(18.71 \mu \mathrm{m})}{I(\mathrm{H} \beta)}\right)+7.051 \\
& -\frac{0.053}{t_{\mathrm{e}}}-0.634 \log t_{\mathrm{e}}
\end{aligned}
$$

and

$$
\begin{aligned}
12+\log \left(\frac{\mathrm{S}^{3+}}{\mathrm{H}^{+}}\right)= & \log \left(\frac{I(10.51 \mu \mathrm{m})}{I(\mathrm{H} \beta)}\right)+6.218 \\
& +\frac{0.098}{t_{\mathrm{e}}}-0.252 \log t_{\mathrm{e}} .
\end{aligned}
$$

Since it is not possible to calculate the electron temperature for most of the objects ( $\sim 90$ per cent) in our IR sample (presented in Table A2), we assumed $T_{\mathrm{e}}=10000 \mathrm{~K}$ that implies a certain amount 
Table 2. Electron temperatures $\left(t_{S 3}\right)$ and sulphur ionic abundances estimated for the Visible and IR samples.

\begin{tabular}{lccccc}
\hline Object & $t_{\mathrm{e}}\left(10^{4} \mathrm{~K}\right)$ & $\log \left(\mathrm{S}^{+} / \mathrm{H}^{+}\right)_{\mathrm{Vis}}$ & $\log \left(\mathrm{S}^{2+} / \mathrm{H}^{+}\right)_{\mathrm{Vis}}$ & $\log \left(\mathrm{S}^{2+} / \mathrm{H}^{+}\right)_{\mathrm{IR}}$ & $\log \left(\mathrm{S}^{3+} / \mathrm{H}^{+}\right)_{\mathrm{IR}}$ \\
\hline N160A1 & 0.92 & -6.24 & -5.20 & -5.31 & -6.03 \\
N160A2 & 0.88 & -6.24 & -5.18 & -5.39 & -6.22 \\
N4A & 0.94 & -6.41 & -5.27 & -5.15 & -5.93 \\
N66 & 1.18 & -6.53 & -5.69 & -5.72 & -6.35 \\
N157-B & 1.29 & -6.09 & -5.49 & -5.30 & -6.57 \\
N88-A & 1.41 & -6.87 & -6.05 & -6.40 & -6.28 \\
N81 & 1.26 & -6.72 & -5.81 & -6.00 & -6.62 \\
Hubble V & 1.09 & -6.68 & - & -5.58 & -6.21 \\
I Zw 36 $^{a}$ & 1.61 & -6.90 & - & -5.81 & -6.08 \\
\hline
\end{tabular}

Note. ${ }^{a}$ See the text for an explanation about the inclusion of these two particular objects.

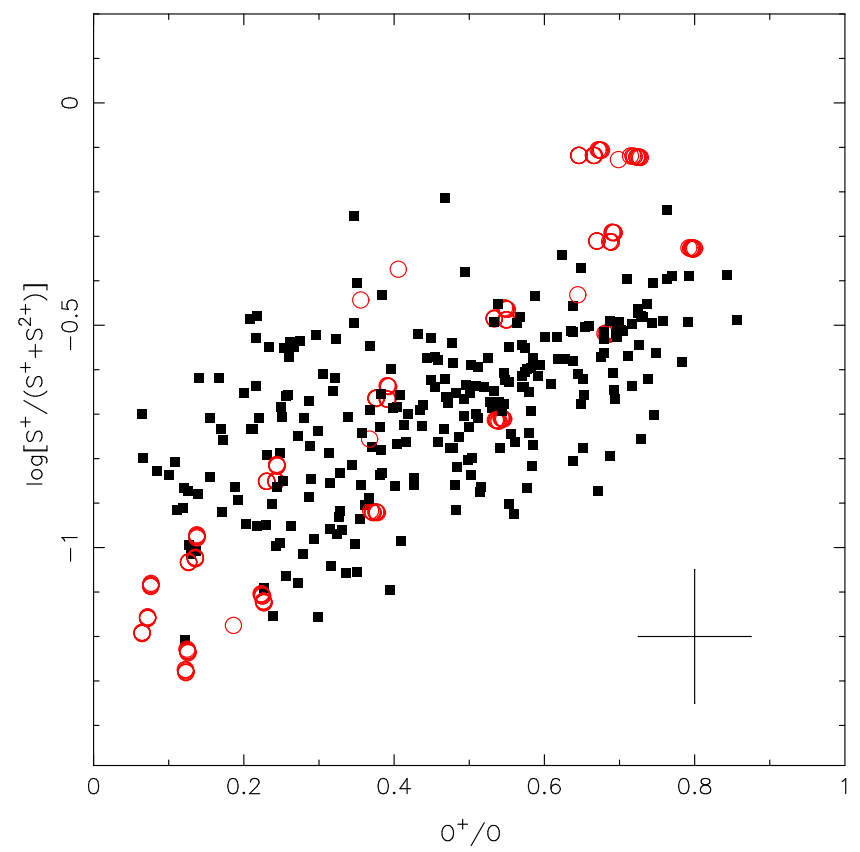

Figure 2. Ionic abundances $\mathrm{S}^{+} /\left(\mathrm{S}^{+}+\mathrm{S}^{2+}\right)$ versus $\mathrm{O}^{+} / \mathrm{O}$. Black squares represent observational ionic determinations computed using the data from Table A1 and the Visible-lines method (see Section 4.2). Open circles represent ionic abundances predicted by our models (see Section 3). The error bar represents typical uncertainties as defined in Section 4.1.

of error. Variations of $\pm 5000 \mathrm{~K}$ in the value of the electron temperature in equations (14) and (15) do the ionic abundance ranges by about \pm 0.1 dex. Moreover, for these objects, we considered the theoretical relation $I(\mathrm{H} \beta) / I\left(\mathrm{H}_{\mathrm{I}} 4.05 \mu \mathrm{m}\right)=12.87$ assuming $N_{\mathrm{e}}=$ $100 \mathrm{~cm}^{-3}$ and $T_{\mathrm{e}}=10000 \mathrm{~K}$ (Osterbrock 1989).

Typical uncertainties in IR estimations are of the order of $0.1 \mathrm{dex}$ and are caused, mainly, by the error in the emission lines (Vermeij $\&$ van der Hulst 2002). Hereafter, we will assume that the ionic abundances calculated from IR-lines method have an uncertainty of 0.10 dex.

\section{RESULTS}

\subsection{Theoretical ICF}

We derived a theoretical ICF for the sulphur based on the photoionization model results described in Section 3. To verify how representative are our models of real $\mathrm{H}$ II regions, in Fig. 2, the ionic abundance ratio $\mathrm{S}^{+} /\left(\mathrm{S}^{+}+\mathrm{S}^{2+}\right)$ against the ionization degree $\mathrm{O}^{+} / \mathrm{O}$

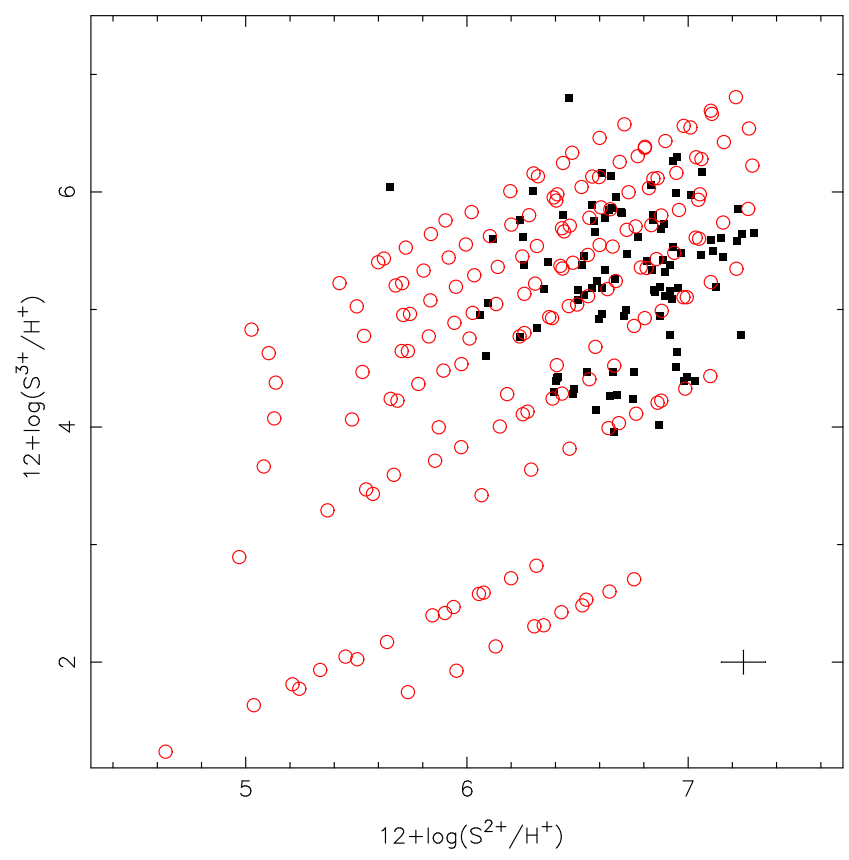

Figure 3. Such as Fig. 2 but for the ionic abundances $\mathrm{S}^{3+} / \mathrm{H}^{+}$versus $\mathrm{S}^{2+} / \mathrm{H}^{+}$computed using the IR sample (Table A2) and the IR-lines method (see Section 4.2).

calculated from the data from Table A1 and using the Visible-lines method are compared with those predicted by the models. The theoretical ionic values considered are the ones weighted over the volume of the hypothetical nebulae. We can see that the models occupy the most part of the region where the observational data are located and they reproduce the tendency of $\mathrm{S}^{+} /\left(\mathrm{S}^{+}+\mathrm{S}^{2+}\right)$ increases with $\mathrm{O}^{+} / \mathrm{O}$. However, there is a region occupied by observational data with $\left[\mathrm{S}^{+} /\left(\mathrm{S}^{+}+\mathrm{S}^{2+}\right)\right] \gtrsim-1$ and $\left(\mathrm{O}^{+} / \mathrm{O}\right) \gtrsim 0.2$ not covered by the models. This seems to be not crucial for this analysis since similar ICFs can be derived from both models and observations, as we are presenting in this paper.

In Fig. 3, the $\mathrm{S}^{3+} / \mathrm{H}^{+}$and $\mathrm{S}^{2+} / \mathrm{H}^{+}$abundances calculated using the IR-lines method and the IR sample and those predicted by the models are shown. Again, we can see that the models cover the region occupied by the observations.

The predictions of the models were used to compute an ICF for the sulphur defined by

$\mathrm{ICF}\left(\mathrm{S}^{+}+\mathrm{S}^{2+}\right)=\frac{\mathrm{S} / \mathrm{H}}{\left(\mathrm{S}^{+}+\mathrm{S}^{2+}\right) / \mathrm{H}^{+}}$, 
Table 3. $\mathrm{O}^{+} / \mathrm{O}$ ionic abundances and direct sulphur ICFs estimations using the Visible-lines and the IR-lines methods.

\begin{tabular}{lcccc}
\hline Object & $\mathrm{O}^{+} / \mathrm{O}$ & Vis & ICF & \\
& & 1.13 & 1.17 \\
\hline N160A1 & 0.256 & 1.08 & 1.13 \\
N160A2 & 0.272 & 1.20 & 1.16 \\
N4A & 0.238 & 1.20 & 1.20 \\
N66 & 0.192 & 1.07 & 1.04 \\
N157-B & 0.404 & 1.60 & 1.98 \\
N88-A & 0.126 & 1.15 & 1.20 \\
N81 & 0.202 & - & 1.21 \\
Hubble V & 0.194 & - & 1.49 \\
I Zw 36 & 0.120 & &
\end{tabular}

where $\mathrm{S} / \mathrm{H}$ is the ratio between the total sulphur and the hydrogen abundances. Assuming the expression suggested by Stasińska (1978a) and presented in equation (2), we found $\alpha=3.27 \pm 0.01$ from a fitting to our model results.

\subsection{Direct ICFs}

When emission lines of the main ionization stages of an element are observed, it is possible to calculate the total abundance of the element and thus, derive an ICF. Therefore, following the methodology presented by Vermeij \& van der Hulst (2002) and Pérez-Montero et al. (2006), we used the Visible and IR samples and the equations presented in Section 4 to derive direct values for the sulphur ICF for the common objects in both samples assuming

$\mathrm{ICF}\left(\mathrm{S}^{+}+\mathrm{S}^{2+}\right)=\frac{\mathrm{S}^{+}+\mathrm{S}^{2+}+\mathrm{S}^{3+}}{\mathrm{S}^{+}+\mathrm{S}^{2+}}$.

This was possible only for nine objects. The $\mathrm{S}^{2+}$ can be estimated using the Visible data and/or using the IR data. Hence, for each object, we have two independent estimations of its sulphur ICF, these two values are named Direct-Vis and Direct-IR ICFs.

The identification of the nine objects for which was possible to compute the ICF by the procedure described above, the electron temperature $\left(t_{S 3}\right)$ and the ionic abundance values are listed in Table A3, while the $\mathrm{O}^{+} / \mathrm{O}$ ratio and the ICF values are presented in Table 3. For Hubble V and IZw 36 were only possible to compute the $\mathrm{S}^{2+}$ ionic abundance via the IR method because the [S III] $\lambda \lambda 9069$, 9532 emission lines are not available in the literature. These are the only two objects in the subsample that do not fulfil the selection criterion to be in the Visible sample but were included here because they contribute to a better estimation of the Direct-IR sulphur ICF. The difference in the $\mathrm{S}^{2+}$ abundances calculated from Visibleand IR-lines methods has an average value of $\sim 0.15 \mathrm{dex}$, with the maximum value of $\sim 0.35 \mathrm{dex}$. In the subsequent paper of this series, we will use photoionization models with abundance variations along the radius of the hypothetical nebula in order to investigate the source of this discrepancy. ${ }^{1}$

In Fig. 4, the direct sulphur ICF values as a function of $\mathrm{O}^{+} / \mathrm{O}$ are plotted together with the corresponding fittings. We found $\alpha=2.76 \pm 0.22$ when $\mathrm{S}^{2+}$ is computed by the IR method and $\alpha=3.08 \pm 0.21$ when the Visible method is considered. We can note in Fig. 4 that the two fits for the estimations based on IR and Visible methods (red and blue lines) seem to be not satisfactory for

\footnotetext{
${ }^{1}$ Similar analysis but applied for neon ionic abundances can be found in Dors et al. (2011).
}

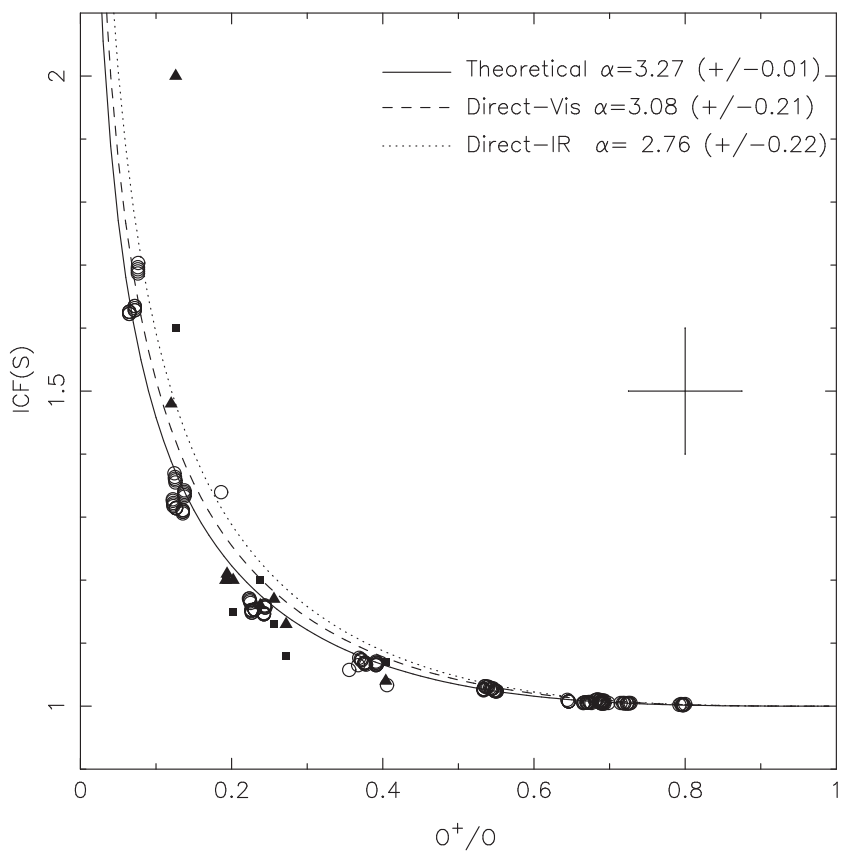

Figure 4. Ionization correction factor for the sulphur versus $\mathrm{O}^{+} / \mathrm{O}$. Squares and triangles represent direct estimations (see Table A3) of the ICF taking into account the $\mathrm{S}^{2+}$ ionic abundance values estimated from the Visible sample (Direc Vis) and the IR sample (Direct IR), respectively. Circles represent estimations of our models. Curves represent the fittings to the equation (2): solid line shows the best fit obtained using our models and dashed and dotted lines the ones obtained using the observational estimations, as indicated. The $\alpha$ values of the best fits are indicated in the legend. The error bars represent typical uncertainties as defined in Section 4.2.

$\mathrm{O}^{+} / \mathrm{O} \lesssim 0.2$, i.e. for the regime of high excitation. Similar result was found by Vermeij \& van der Hulst (2002). A larger number of direct ICF estimations for objects with high excitation is clearly need to improve the results for this regime.

The error in the Direct-ICF value is due to the uncertainties of ionic abundance determinations $\left(\mathrm{S}^{+}, \mathrm{S}^{2+}, \mathrm{O}^{+}\right.$, and $\left.\mathrm{O}^{2+}\right)$ and due to the discrepancy between the abundance of $\mathrm{S}^{2+}$ calculated via Visible and IR methods (Dors et al. 2013; Vermeij \& van der Hulst 2002). Based on the results of Vermeij \& van der Hulst (2002), we assumed an average error of 0.2 for the Direct-ICF and 0.15 for $\mathrm{O}^{+} / \mathrm{O}$, obtained from ionic estimations of Kennicutt et al. (2003). These uncertainties yield an error in the total sulphur abundance of only $\sim 10$ per cent.

\section{DISCUSSION}

In their pioneer paper, Peimbert \& Costero (1969) obtained photoelectric observations of the $\mathrm{H}$ II regions Orion, M 8 and $\mathrm{M} 17$ and suggested that the total abundance of the sulphur can be obtained by using an ICF defined by the ionic abundance ratio $\left(\mathrm{O}^{+}+\mathrm{O}^{2+}\right) / \mathrm{O}^{+}$. This empirical approach is based on the similarity between the ionization potentials of $\mathrm{S}^{2+}$ and $\mathrm{O}^{+}$. During the next decades, sulphur ICFs had been mainly derived from the analytical expression suggested by Stasińska (1978a), and the $\alpha$ value of this original prescription have been largely discussed. For example, data obtained with the Infrared Space Observatory by Vermeij et al. (2002) became, possibly, the first test for the $\alpha$ value, since direct estimations of sulphur ICFs were possible. These authors showed that an $\alpha$ value equal to 2, as suggested by French (1981), overpredicts the 

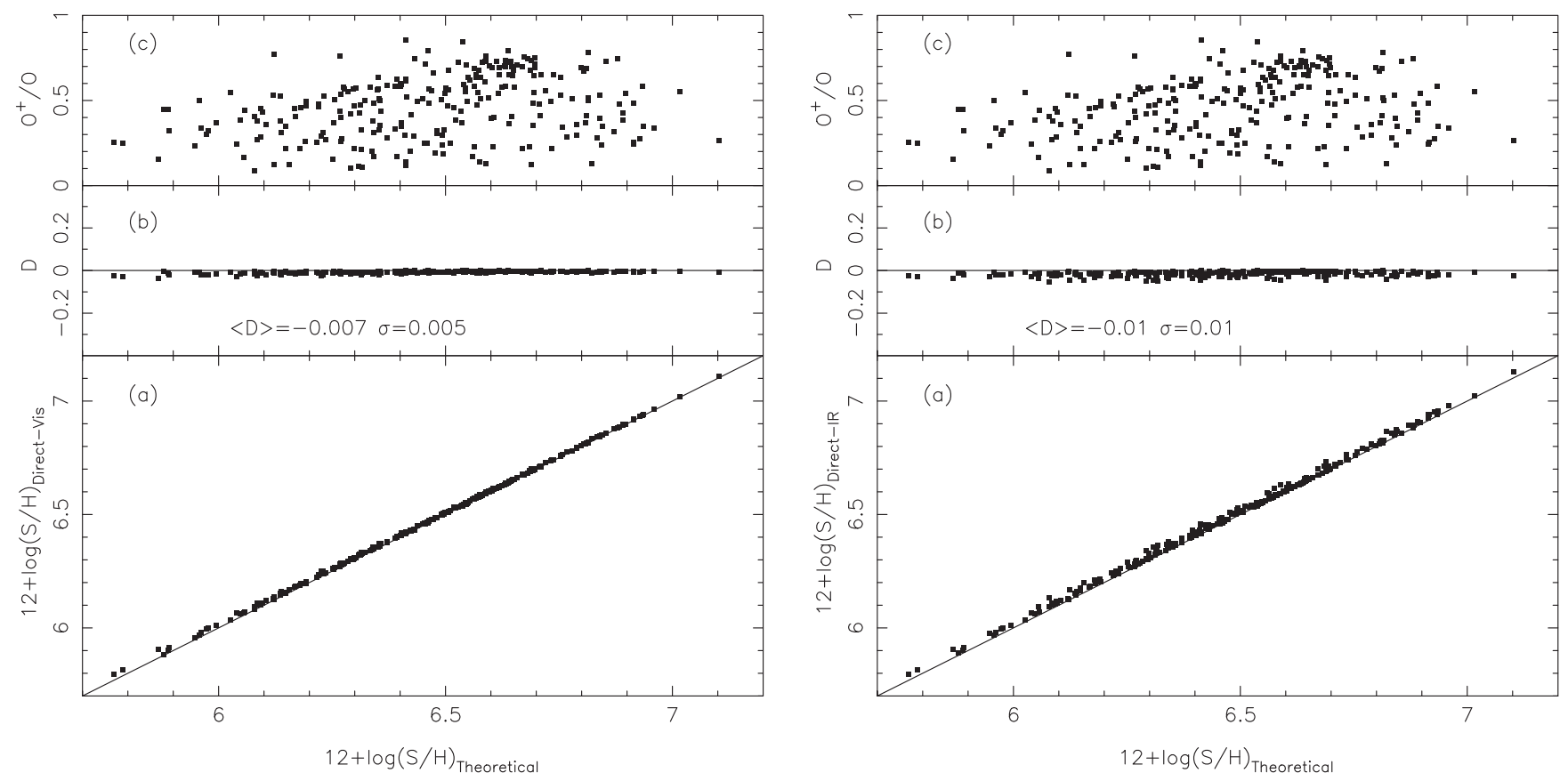

Figure 5. Panel a: comparison between the S/H total abundances obtained for the objects in the Visible sample applying the theoretical ICF and, Direct-Vis (left plot) and Direct-IR (right plot) ICFs, as indicated. Panel b: differences between the estimations using the considered ICFs with their average value ( $\langle\mathrm{D}\rangle$ ) and its dispersion $(\sigma)$ indicated. Panel $\mathrm{c}: \mathrm{O}^{+} / \mathrm{O}$ ratio for each estimation.

$\mathrm{S}^{3+}$ ionic abundance, in concordance with the result previously obtained by Garnett (1989). From their observational data, Vermeij \& van der Hulst (2002) concluded that $\alpha=3$ is a more reliable value, at least for $\mathrm{O}^{+} / \mathrm{O}>0.2$.

Despite ICFs could be obtained from direct calculation of ionic abundances (Vermeij \& van der Hulst 2002) or even from ionization potential considerations (e.g. Peimbert \& Costero 1969; French 1981), ICFs based on grids of photoionization models of nebulae are more reliable because all ionization stages of a given ion as well as several physical processes (e.g. charge transfer reactions) are taken into account in the calculations (Stasińska 2002). In this work, we built a grid of photoionization models assuming a large range of nebular parameters (e.g. $Z, U, \mathrm{~S} / \mathrm{O}$ ) and derived a theoretical sulphur ICF. Based on the agreement between the model predictions and data of a large sample of objects, we suggested an $\alpha$ value of $3.27 \pm 0.01$ in the Stasińska formulae. This value is somewhat higher than the one derived by Vermeij \& van der Hulst (2002), but it is in consonance with the one derived through direct ionic estimations $(\alpha=3.08 \pm 0.21)$ based mainly on the Visible-line method (Direct-Vis ICF).

With the aim to compare the $\mathrm{S} / \mathrm{H}$ total abundances yielded by the use of different ICFs, we considered the relation:

$\frac{\mathrm{S}}{\mathrm{H}}=\mathrm{ICF}(\mathrm{S}) \times \frac{\mathrm{S}^{+}+\mathrm{S}^{2+}}{\mathrm{H}^{+}}$,

using the $\mathrm{S}^{+}$and $\mathrm{S}^{2+}$ ionic abundances estimated for the objects in our Visible sample via the Visible-lines method. First, we compared the $\mathrm{S} / \mathrm{H}$ abundances derived through the theoretical ICF $(\alpha=3.27$ $\pm 0.01)$, with those derived using the Direct-Vis ICF $(\alpha=3.08$ $\pm 0.21)$ and the Direct-IR ICF $(\alpha=2.76 \pm 0.22)$. In panels a of Fig. 5, these comparisons are shown. In this figure, we also plotted the differences (D) between the $\mathrm{S} / \mathrm{H}$ total abundances estimations (panels b) and the $\mathrm{O}^{+} / \mathrm{O}$ ratio (panels c). It can be seen that the theoretical ICF yields $\mathrm{S} / \mathrm{H}$ total abundances in excellent agreement with those given by the Direct-Vis and Direct-IR ICFs, with an average difference $\langle\mathrm{D}\rangle \approx 0.00$ and dispersions of 0.005 and 0.01 dex, respectively, independently of the ionization degree that is sampled by the $\mathrm{O}^{+} / \mathrm{O}$ ratio.

Secondly, we also compare the $\mathrm{S} / \mathrm{H}$ total abundance estimations based on our theoretical ICF with the ones obtained using some ICFs proposed in the literature. In what follows, a brief description of these ICFs is presented.

(i) Kennicutt et al. ICF- Kennicutt et al. (2003) proposed to use $\alpha=2.5$ for typical $\mathrm{H}_{\text {II }}$ regions. This is an average value obtained from the photoionization models grid calculated by Garnett (1989). The same $\alpha$ value was obtained by Pérez-Montero et al. (2006) from optical and IR data.

(ii) Izotov et al. ICF- Izotov et al. (2006a) used a grid of photoionization models by Stasińska \& Izotov (2003) built assuming spectral energy distributions calculated with the STARBURST99 (Leitherer et al. 1999) and stellar atmosphere models by Smith, Norris \& Crowther (2002) to derive an expression for the sulphur ICF. These authors derived ICFs considering three metallicity regimes: low $[12+\log (\mathrm{O} / \mathrm{H})<7.6]$, intermediate $[7.6<12+\log (\mathrm{O} / \mathrm{H})<$ 8.2] and high $[12+\log (\mathrm{O} / \mathrm{H})>8.2]$, which are given by

$$
\begin{aligned}
\operatorname{ICF}(\mathrm{S}) & =0.121 x+0.511+0.161 / x, \text { low } Z, \\
& =0.155 x+0.849+0.062 / x, \text { inter. } Z, \\
& =0.178 x+0.610+0.153 / x \quad \operatorname{high} Z,
\end{aligned}
$$

where $x=\mathrm{O}^{+} / \mathrm{O}$.

(iii) Thuan et al. ICF- Thuan et al. (1995), who used the results of photoionization models grid built by Stasińska (1990) and NLTE atmosphere models by Mihalas (1972), derived

$\mathrm{ICF}=[0.013+x[5.10+x(-12.78+x(14.77-6.11 x))]]^{-1}$.

(iv) Kwitter \& Henry ICF- Kwitter \& Henry (2001) built a grid of photoionization models considering a blackbody as the ionizing 

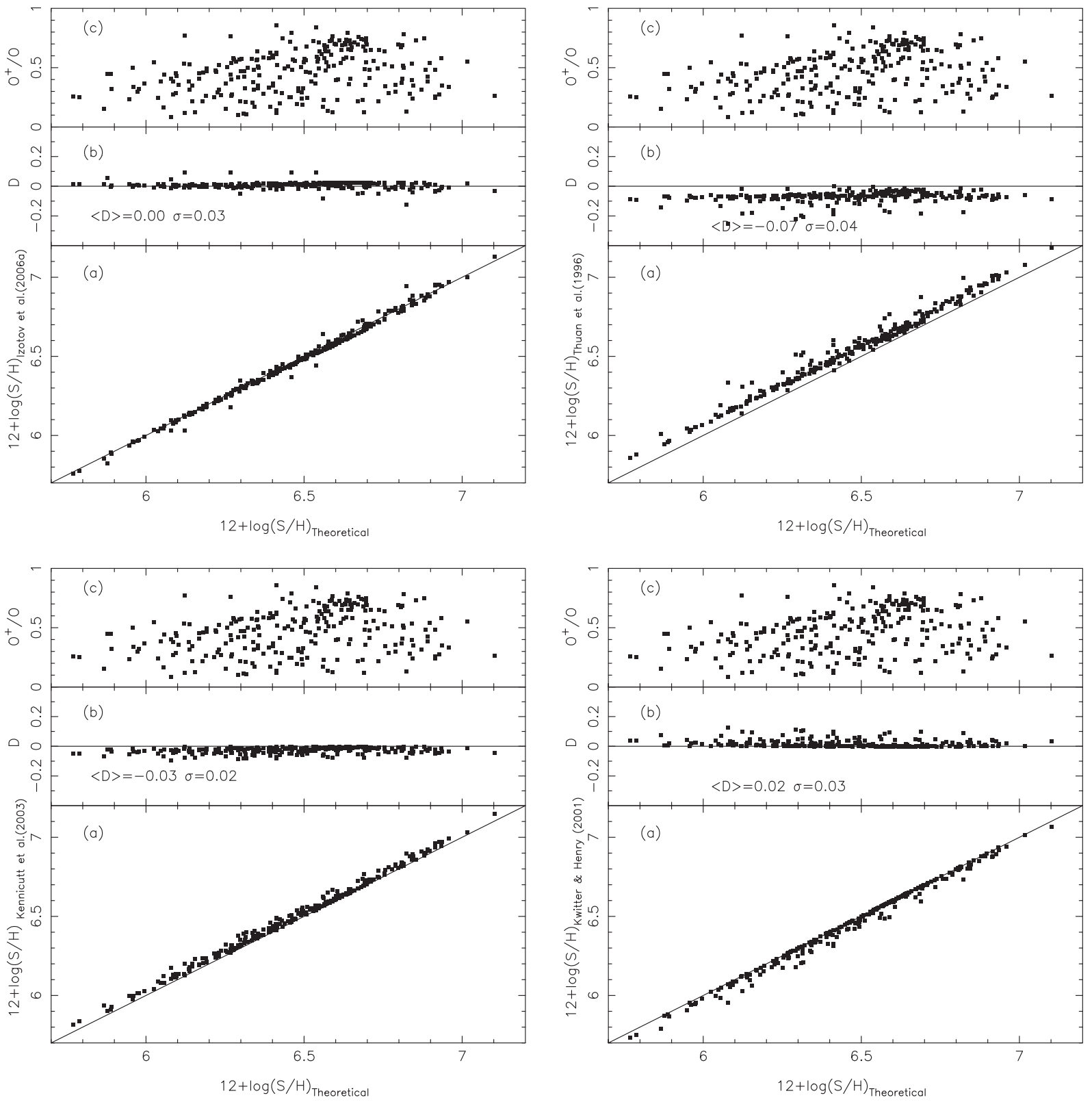

Figure 6. Idem Fig. 5 for different ICFs from the literature, as indicated.

source in order to derive sulphur ICFs for planetary nebulae that, in principle, it can be employed for $\mathrm{H}_{\text {II }}$ regions. These authors proposed

$\operatorname{ICF}(\mathrm{S})=e^{-0.017+(0.18 \beta)-\left(0.11 \beta^{2}\right)+\left(0.072 \beta^{3}\right)}$,

where $\beta=\log \left(\mathrm{O} / \mathrm{O}^{+}\right)$.

(v) Delgado-Inglada et al. ICF - Delgado-Inglada et al. (2014) computed a large grid of photoionization models in order to derive new formulae for ICFs of several elements to be applied in studies of planetary nebulae. The expression derived by these authors to calculate the total abundance $\mathrm{S} / \mathrm{H}$ can be to write in the form

$\frac{\mathrm{S}}{\mathrm{H}}=\mathrm{ICF}(\mathrm{S}) \times \frac{\mathrm{S}^{+}+\mathrm{S}^{2+}}{\mathrm{O}^{+}} \times \frac{\mathrm{O}}{\mathrm{H}}$,

where

$\mathrm{ICF}(\mathrm{S})=\frac{-0.02-0.03 w-2.31 w^{2}+2.19 w^{3}}{0.69+2.09 w-2.69 w^{2}}$, and

$w=\mathrm{O}^{2+} / \mathrm{O}$.

In Figs 6 and 7 (panels a), a comparison between S/H total abundance estimations based on our theoretical ICF and those from the literature are shown. In these figures, we also show the difference (D) between these estimations (panels b) and the $\mathrm{O}^{+} / \mathrm{O}$ ratio (panels c). Taking into account the typical errors found in the S/H total abundance estimations (see e.g. Hägele et al. 2008) and the dispersion $(\sigma)$ of the average differences $(\langle\mathrm{D}\rangle)$, it might seem that the different $\mathrm{S} / \mathrm{H}$ estimations are in agreement. However, with exception of the ICF of Izotov et al. (2006a), there are clear systematic differences between the values derived through the use of our theoretical ICF and from the other ICFs. Moreover, difference in $\mathrm{S} / \mathrm{H}$ abundances obtained from distinct ICFs can be not negligible when only an individual object is considered. In fact, we noted that 


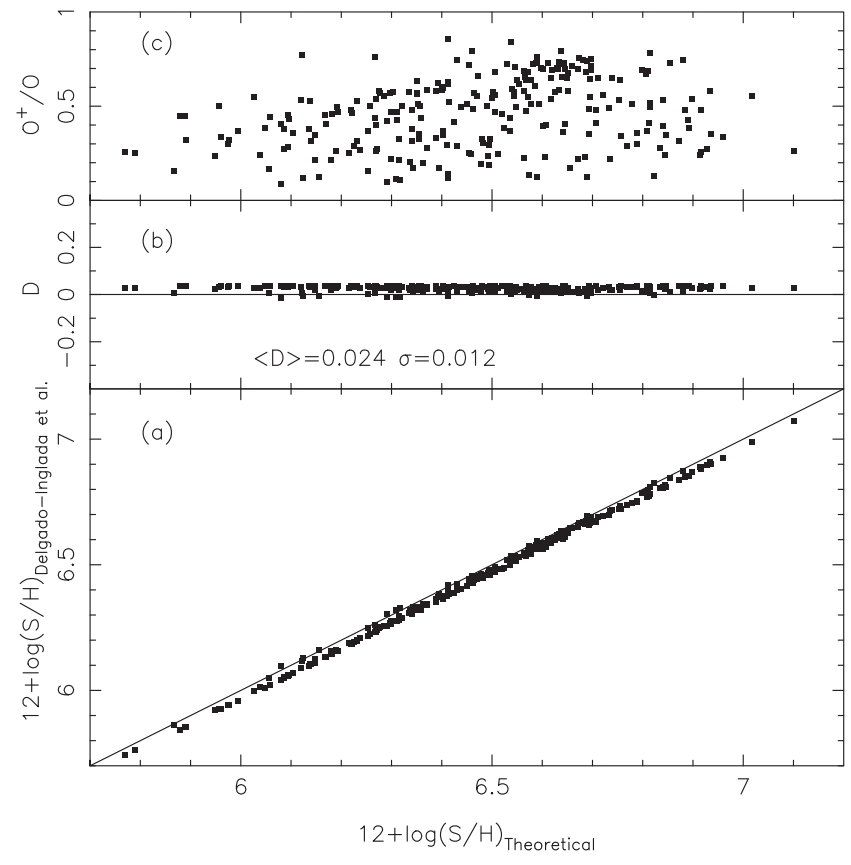

Figure 7. Idem Fig. 5 for the ICF proposed by Delgado-Inglada, Morisset \& Stasińska (2014)

it could reach up to about 0.3 dex for the low-metallicity regime (see Fig. 5).

Concerning the ratio between sulphur and oxygen abundances, several studies have addressed the investigation about the variation of S/O with $\mathrm{O} / \mathrm{H}$ in individual galaxies (e.g. Vílchez et al. 1988; Christensen, Petersen \& Gammelgaard 1997; Garnett et al. 1997; Vermeij \& van der Hulst 2002; Kennicutt, Bresolin \& Garnett 2003; López-Sánchez \& Esteban 2009; Berg et al. 2013; Skillman et al. 2013; Croxall et al. 2015) or in a general context (e.g. Izotov et al. 1997; Henry \& Worthey 1999; Kehrig et al. 2006; Pérez-Montero et al. 2006; Hägele et al. 2008, 2012; Guseva et al. 2011). Most of these results indicate that the ratio S/O appears to be constant with the metallicity, which argues that either these elements are produced by massive stars within a similar mass range or by stars with a distinct mass interval but being formed with an universal IMF (Henry \& Worthey 1999). However, when a large sample of data is considered, the dispersion found is very large and the assumption of a constant S/O ratio is questionable (Kehrig et al. 2006; PérezMontero et al. 2006; Hägele et al. 2008, 2012). Therefore, with the goal of studying the relation of the S/O ratio with the metallicity (traced by the $\mathrm{O} / \mathrm{H}$ abundance), we used the data listed in Table A1 and all the ICFs considered in this work to calculate S/O and $\mathrm{O} / \mathrm{H}$ ratios via the Visible-lines method. The Direct-Vis ICF was not considered since its $\alpha$ value is very similar to that of the theoretical one. In Fig. 8, only the estimations obtained from the theoretical ICF is shown. For estimations from other ICFs (not shown), similar results were obtained. The solar values $\log (\mathrm{S} / \mathrm{O})_{\odot}=-1.43$ and $12+\log (\mathrm{O} / \mathrm{H})_{\odot}=8.69$ derived using the sulphur abundance from Grevesse \& Sauval (1998) and the oxygen one from Allende Prieto et al. (2001) are also indicated. We can see in this figure that most of the objects present subsolar $\mathrm{S} / \mathrm{O}$ and $\mathrm{O} / \mathrm{H}$ abundance ratios. Interestingly, for the extreme low-metallicity regime, some of the objects reach very high S/O abundance ratios. Since the dispersion is high and the number of objects is much lower than for the high-metallicty regime, more data are needed to confirm this result.

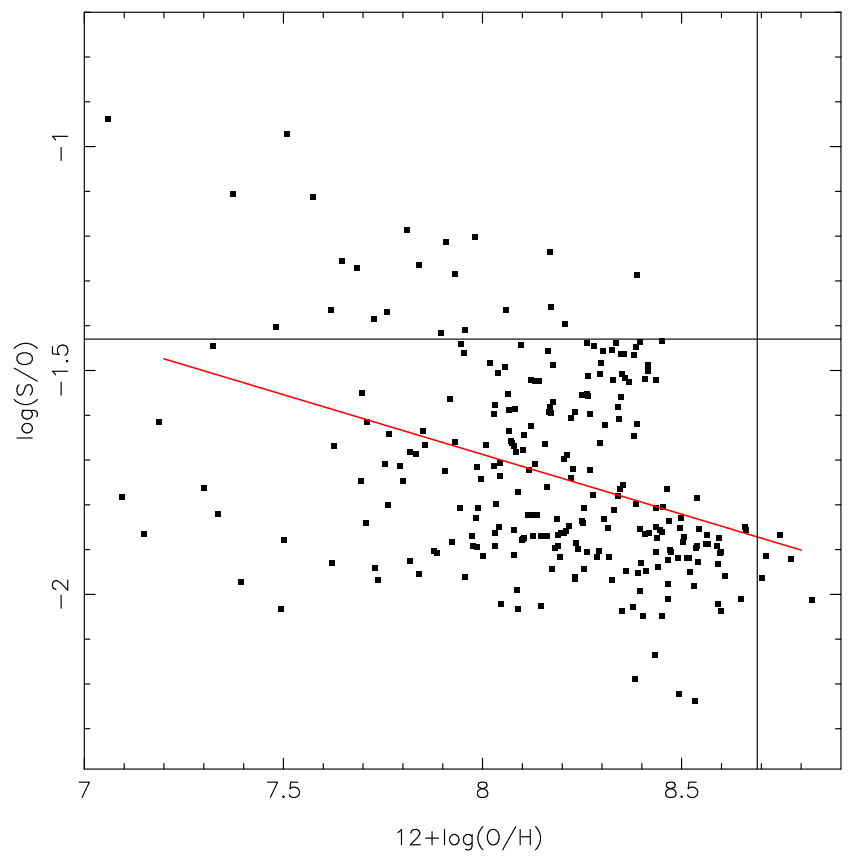

Figure 8. Relation between $\log (\mathrm{S} / \mathrm{O})$ and $12+\log (\mathrm{O} / \mathrm{H})$ ratios using the Visible-lines method and our theoretical ICF. Black lines represent the solar $\mathrm{S} / \mathrm{O}$ and $\mathrm{O} / \mathrm{H}$ abundance ratios derived using the oxygen abundance from Allende Prieto et al. (2001) and sulphur abundance from Grevesse \& Sauval (1998). Red lines represent linear regressions to the observational estimations. Coefficients of this regression are given in Table A4.

We also performed a fit to these data, assuming a linear regression without taking into account the individual errors. In Table A4, the coefficients of the fittings, and the linear regressions considering all ICFs are listed. We found that the S/O ratio decreases with metallicity, yielding a mean slope of about -0.27 with all the fitted slopes in agreement within the estimated errors. We also obtained the average values for $\log (\mathrm{S} / \mathrm{O})$ estimated via the different ICFs and considering the three different metallicity regimes. These values and the number of objects used to calculate them are also listed in Table A4. Considering all the metallicity regimes together and all the considered ICFs, we found an average $\langle\log (\mathrm{S} / \mathrm{O})\rangle=-1.72 \pm$ 0.03 . Despite the dispersion, when low-, intermediate- and highmetallicities regimes are separately considered, we note a decrease in S/O when the metallicity increases. For low- and high-metallicity regimes, we derived mean values of $\langle\log (\mathrm{S} / \mathrm{O})\rangle-1.53 \pm 0.05$ and $-1.78 \pm 0.02$, respectively. Similar results were also derived by Díaz et al. (1991), Vílchez et al. (1988) for M51 and M33 galaxies and by Shaver et al. (1983) for Milky Way.

\section{CONCLUSIONS}

We built a grid of photoionization models combined with stellar population synthesis models to derive ICFs for the sulphur. The reliability of these ICFs was obtained from the agreement between ionic abundances predicted by the models and those calculated through optical and IR spectroscopic data of star-forming regions with a very wide range in metallicity $(7.0 \lesssim 12+\log (\mathrm{O} / \mathrm{H}) \lesssim 8.8)$ and ionization degree $\left(0.1 \lesssim \mathrm{O}^{+} / \mathrm{O} \lesssim 0.9\right)$. From our results, we suggest $\alpha=3.27 \pm 0.01$ to be used in the classical Stasińska formula. This $\alpha$ value is in consonance with the one derived from direct estimations based on spectroscopic data of a small sample of objects. A comparison of the $\mathrm{S} / \mathrm{H}$ total abundance derived by us for 
Table 4. Coefficients of the linear regressions $\log (\mathrm{S} / \mathrm{O})=\mathrm{a} \times[12+\log (\mathrm{O} / \mathrm{H})]+\mathrm{b}$ fitted to the data plotted in Fig. 8 taking into account each of the considered ICF. Mean values for the abundance ratio $\log (\mathrm{S} / \mathrm{O})$ considering all the metallicity range as well as the corresponding ones for the low $[12+\log (\mathrm{O} / \mathrm{H})<7.6]$, intermediate $[7.6<12+\log (\mathrm{O} / \mathrm{H})<8.2]$ and high $[12+\log (\mathrm{O} / \mathrm{H})>8.2]$ metallicity regimes, and the number of objects used in these calculations are shown.

\begin{tabular}{|c|c|c|c|c|c|c|}
\hline \multirow[b]{2}{*}{ Number objects } & \multirow[b]{2}{*}{$\mathrm{a}$} & \multirow[b]{2}{*}{$\mathrm{b}$} & \multicolumn{4}{|c|}{$\begin{array}{c}\langle\log (\mathrm{S} / \mathrm{O})\rangle \\
\text { Metallicity regime }\end{array}$} \\
\hline & & & $\begin{array}{l}\text { All } \\
261\end{array}$ & $\begin{array}{c}\text { Low } \\
14\end{array}$ & $\begin{array}{c}\text { Intermediate } \\
114\end{array}$ & $\begin{array}{c}\text { High } \\
133\end{array}$ \\
\hline \multicolumn{7}{|l|}{$\mathrm{ICF}$} \\
\hline Direct IF & $-0.28 \pm 0.04$ & $0.60 \pm 0.33$ & $-1.71 \pm 0.22$ & $-1.52 \pm 0.36$ & $-1.66 \pm 0.20$ & $-1.78 \pm 0.20$ \\
\hline Theoretical & $-0.26 \pm 0.04$ & $0.45 \pm 0.33$ & $-1.73 \pm 0.22$ & $-1.55 \pm 0.38$ & $-1.68 \pm 0.20$ & $-1.79 \pm 0.19$ \\
\hline Kennicut et al. & $-0.30 \pm 0.04$ & $0.72 \pm 0.33$ & $-1.70 \pm 0.22$ & $-1.50 \pm 0.35$ & $-1.64 \pm 0.20$ & $-1.77 \pm 0.20$ \\
\hline Izotov et al. & $-0.28 \pm 0.04$ & $0.60 \pm 0.33$ & $-1.73 \pm 0.22$ & $-1.53 \pm 0.31$ & $-1.68 \pm 0.20$ & $-1.80 \pm 0.20$ \\
\hline Thuan et al. & $-0.32 \pm 0.04$ & $0.94 \pm 0.33$ & $-1.66 \pm 0.23$ & $-1.44 \pm 0.32$ & $-1.59 \pm 0.20$ & $-1.73 \pm 0.20$ \\
\hline Kwitter \& Henry & $-0.23 \pm 0.04$ & $0.13 \pm 0.34$ & $-1.75 \pm 0.22$ & $-1.59 \pm 0.41$ & $-1.71 \pm 0.21$ & $-1.79 \pm 0.19$ \\
\hline Delgado-Inglada et al. & $-0.26 \pm 0.04$ & $0.41 \pm 0.33$ & $-1.75 \pm 0.22$ & $-1.56 \pm 0.38$ & $-1.71 \pm 0.21$ & $-1.81 \pm 0.19$ \\
\hline
\end{tabular}

the objects in our Visible sample and considering different ICFs proposed in the literature was performed. Although, in average, the differences between these determinations are similar to the uncertainties in the $\mathrm{S} / \mathrm{H}$ estimations, we noted that it could reach up to about 0.3 dex for the low-metallicity regime. Finally, the highest $\mathrm{S} / \mathrm{O}$ abundance ratios are derived for objects with extreme lowmetallicity values. Indeed, a tendency of the S/O ratio to decrease with the metallicity was found, independently of the considered ICF.

\section{ACKNOWLEDGEMENTS}

We are very grateful to the anonymous referee for his/her complete and deep revision of our manuscript, and very useful comments and suggestions that helped us to substantially clarify and improve our work and to Dr Gary Ferland for making available the CLOUDY programme.

\section{REFERENCES}

Aggarwal K. M., Keenan F. P., 1999, ApJS, 123, 311

Allende Prieto C., Lambert D. L., Asplund M., 2001, ApJ, 556, 63

Arnett W. D., 1978, ApJ, 219, 1008

Berg D. A., Skillman E. D., Garnett D. R., Croxall K. V., Marble A. R., Smith J. D., Gordon K., Kennicutt R. C., Jr 2013, ApJ, 775, 128

Bernard-Salas J. et al., 2009, ApJS, 184, 230

Bresolin F., Kennicutt R. C., Garnett D. R., 1999, ApJ, 510, 104

Bresolin F., Schaerer D., González Delgado R. M., Stasińska G., 2005, A\&A, 441, 981

Bresolin F., Gieren W., Kudritzki R.-P., Pietrzyński G., Urbaneja M. A., Carraro G., 2009, ApJ, 700, 309

Castelli F., Kurucz R. L., 2004, preprint (arXiv: astro-ph/0405087)

Christensen T., Petersen L., Gammelgaard P., 1997, A\&A, 322, 41

Croxall K. V., Pogge R. W., Berg D., Skillman E. D., Moustakas J., 2015, ApJ, 808, 42

Delgado-Inglada G., Morisset C., Stasińska G., 2014, MNRAS, 440, 536

Dennefeld M., Stasińska G., 1983, A\&A, 118, 234

Díaz A. I., Terlevich E., Vílchez J. M., Pagel B. E. J., Edmunds M. G., 1991, MNRAS, 253, 245

Dors O. L., Krabbe A. C., Hägele G. F., Pérez-Montero E., 2011, MNRAS, 415,3616

Dors O. L. et al., 2013, MNRAS, 432, 2512

Esteban C., García-Rojas J., Carigi L., Peimbert M., Bresolin F., LópezSnchez A. R., Mesa-Delgado A., 2014, MNRAS, 443, 624

Ferland G. J. et al., 2013, Rev. Mex. Astron. Astrofis., 49, 137
Freitas-Lemes P., Rodrigues I., Dors O. L. Jr., Fáundez-Abans M., PérezMontero E., 2014, MNRAS, 441, 1086

French H. B., 1981, ApJ, 246, 434

Garnett D. R., 1989, ApJ, 345, 282

Garnett D. R., Shields G. A., Skillman E. D., Sagan S. P., Dufour R. J., 1997, ApJ, 489, 63

Giveon U., Sternberg A., Lutz D., Feuchtgruber H., Paudrach A. W. A., 2002, ApJ, 566, 880

González-Delgado R. M., Perez E., Diaz A. I., Garcia-Vargas M. L., Terlevich E., Vilchez J. M., 1995, ApJ, 439, 604

Grevesse N., Sauval. A. J., 1998, Space Sci. Rev., 85, 161

Guseva N. G., Izotov Y. I., Stasińska G., Fricke K. J., Henkel C., Papaderos P., 2011, A\&A, 529, 149

Hägele G. F., Pérez-Montero E., Díaz A. I., Terlevich E., Terlevich R., 2006, MNRAS, 372, 293

Hägele G. F., Díaz A. I., Terlevich E., Terlevich R., Prez-Montero E., Cardaci M. V., 2008, MNRAS, 383, 209

Hägele G. F., García-Benito R., Prez-Montero E., Daz A. I., Cardaci M. V., Firpo V., Terlevich E., Terlevich R., 2011, MNRAS, 414, 272

Hägele G. F., Firpo V., Bosch G., Díaz Á. I., Morrell N., 2012, MNRAS, 422,3475

Helmboldt J. F., Walterbos R. A. M., Bothun G. D., O'Neil K, 2005, ApJ, 630,824

Henry R. C. B, Worthey G., 1999, PASP, 111, 919

Izotov Y. I., Thuan T. X., Lipovetsky V. A., 1994, ApJ, 435, 647

Izotov Y. I., Lipovetsky V. A., Chaffee F. H., Foltz C. B., Guseva N. G., Kniazev A. Y., 1997, ApJ, 476, 698

Izotov Y. I., Stasińska G., Meynet G., Guseva N. G., Thuan T. X., 2006a, A\&A, 448, 955

Izotov Y. I., Schaerer D., Blecha A., Royer F., Guseva N. G., North P., 2006b, A\&A, 459, 71

Johnson C. T., Kingston A. E., Dufton P. L., 1986, 220, 155

Kehrig C., Vílchez J. M., Telles E., Cuisinier F., Pérez-Montero E., 2006, A\&A, 457, 477

Kennicutt R. C., Bresolin F., Garnett D. R., 2003, ApJ, 591, 801

Kewley L. J., Dopita M. A., Sutherland R. S., Heisler C. A., Trevena J., 2001, ApJ, 556, 121

Kewley L. J., Jansen R. A., Geller M. J., 2005, PASP, 117, 227

Krabbe A. C., Rosa D. A., Dors O. L., Pastoriza M. G., Winge C., Hägele G. F., Cardaci M. V., Rodrigues I., 2014, MNRAS, 437, 1155

Kwitter K. B., Henry R. B. C., 2001, ApJ, 562, 804

Lebouteiller V., Bernard-Salas J., Brandl B., Whelan D. G., Wu Y., Charmandaris V., Devost D., Houck J. R., 2008, ApJ, 680, 398

Leitherer C. et al., 1999, ApJ, 123, 3

López-Hernández J., Terlevich E., Terlevich R., Rosa-González D., Díaz Á., Garca-Benito R., Vlchez J., Hägele G., 2013, MNRAS, 430,472

López-Sánchez A. R., Esteban C., 2009, A\&A, 508, 615 
Luridiana V., Morisset C., Shaw R. A., 2015, A\&A, 573A, 42

Mihalas D., 1972, Non-LTE model atmospheres for B and O stars, NCARTN/STR-76, Boulder

Nollenberg J., Skillman E., Garnett D. R., Dinerstein H., 2002, ApJ, 581, 1002

Osterbrock D. E., 1989, Astrophysics of Gaseous Nebulae and Active Galactic Nuclei. University Science Books, Sausalito, CA

Pauldrach A. W. A., Hoffmann T. L., Lennon M., 2001, A\&A, 375, 161

Peeters E. et al., 2002, A\&A, 381, 571

Peimbert M., Costero R., 1969, Bol. Obs. Tonantzinka Tacubaya, 5, 3

Pérez-Montero E., 2014, MNRAS, 441, 2663

Pérez-Montero E., Contini T., 2009, MNRAS, 398, 949

Pérez-Montero E., Díaz A. I., 2003, MNRAS, 346, 105

Pérez-Montero E., Díaz A. I., 2005, 361, 1063

Pérez-Montero E., Díaz A. I., Vílchez J. M., Kehrig C., 2006, A\&A, 449, 193

Podobedova L. I., Kelleher D. E., Wiese W. L., 2009, J. Phys. Chem. Ref. Data, 38, 171

Pradhan A. K., Montenegro M., Nahar S. N., Eissner W., 2006, MNRAS, 366, L6

Rosa D. A., Dors O. L., Krabbe A. C., Hägele G. F., Cardaci M. V., Pastoriza M. G., Rodrigues I., Winge C., 2014, MNRAS, 444, 2005

Rubin R. H., Simpson J. P., Lord S. D., Colgan S. W. J., Erickson E. F., Haas M. R., 1994, ApJ, 420, 772

Rubin R. H. et al., 2008, MNRAS, 387, 45

Russell S. C., Dopita M. A., 1990, ApJS, 74, 93

Sánchez S. F. et al., 2015, A\&A, 574, 47

Shaver P. A., McGee R. X., Newton L. M., Danks A. C., Pottasch S. R., 1983, MNRAS, 204, 53

Shure M. A., Herter T., Houck J. R., Gull G. E., Briotta D. A., Jr, Forrest W. J., McCarthy J. F., 1983, ApJ, 270, 645

Simpson J. P., Witterborn F. C., Price S. D., Cohen M., 1998, ApJ, 508, 268
Simpson J. P., Cotera A. S., Burton M. G., Cunningham M. R., Lo N., Bains I., 2012, MNRAS, 419, 211

Skillman E. D., Kennicutt R. C., 1993, ApJ, 411, 655

Skillman E. D. et al., 2013, AJ, 146, 3

Smith L. J., Norris R. P. F., Crowther P. A., 2002, MNRAS, 337, 1309

Stasińska G., 1978, A\&A, 66, 257

Stasińska G., 1978, A\&AS, 32, 429

Stasińska G., 1990, A\&AS, 83, 501

Stasińska G., 2002, preprint (arXiv:astro-ph/0207500)

Stasińska G., Izotov Y. I., 2003, A\&A, 397, 71

Storey P. J., Hummer D. G., 1995, MNRAS, 272, 41

Storey P. J., Zeippen C. J., 2000, MNRAS, 312, 813

Tayal S. S., 2000, ApJ, 530, 1091

Tayal S. S., Gupta G. P., 1999, ApJ, 526, 544

Tayal S. S., Zatsarinny O., 2010, ApJS, 188, 32

Thuan T. X., Izotov Y. I., Lipovetsky V. A., 1995, ApJ, 445, 108

van Hoof P. A. M., Weingartner J. C., Martin P. G., Volk K., Ferland G. J., 2001, in Ferland G., Savin D., eds, ASP Conf Ser. Vol. 247, Challenges of Photoionized Plasmas. Astron. Soc. Pac., San Francisco, p. 363

Vermeij R., van der Hulst J. M., 2002, A\&A, 391, 1081

Vermeij R., Damour F., van der Hulst J. M., Baluteau J.-P., 2002, A\&A, 390, 649

Vila-Costas M. B., Edmunds M. G., 1993, MNRAS, 265, 199

Vílchez J. M., Iglesias-Páramo J., 2003, ApJS, 145, 225

Vílchez J. M., Pagel B. E. J., Díaz A. I., Terlevich E., Edmunds M. G., 1988, MNRAS, 235, 633

Walterbos R. A. M., 1998, PASA, 15, 99

Woosley S. E., Weaver T. A., 1995, ApJS, 101, 181

York D. G. et al., 2000, AJ, 120, 1579

Zeippen C. J., 1982, MNRAS, 198, 111

Zurita A., Bresolin F., 2012, MNRAS, 427, 1463 
APPENDIX A

Table A1. Dereddened line fluxes (relative to $\mathrm{H} \beta=100.0)$ compiled from the literature. The intensity of the line [O II] $\lambda 3727$ represents the sum of the lines [O II] $] \lambda 3726$ + 29. References - Data compiled by (1) Kennicutt et al. (2003), (2) Vermeij et al. (2002), (3) Hägele et al. (2008), (4) Bresolin et al. (2009), (5) Vílchez \& Iglesias-Páramo (2003) (6) Hägele et al. (2006), (7) Hägele et al. (2011), (8) Izotov et al. (2006b), (9) Guseva et al. (2011), (10) Garnett et al. (1997), (11) Vílchez et al. (1988), (12) Skillman et al. (2013), (13) López-Hernández et al. (2013), (14) Zurita \& Bresolin (2012), (15) Pérez-Montero \& Díaz (2003), (16) González-Delgado et al. (1995), (17) Skillman \& Kennicutt (1993), (18) Russell \& Dopita (1990), (19) Hägele et al. (2012).

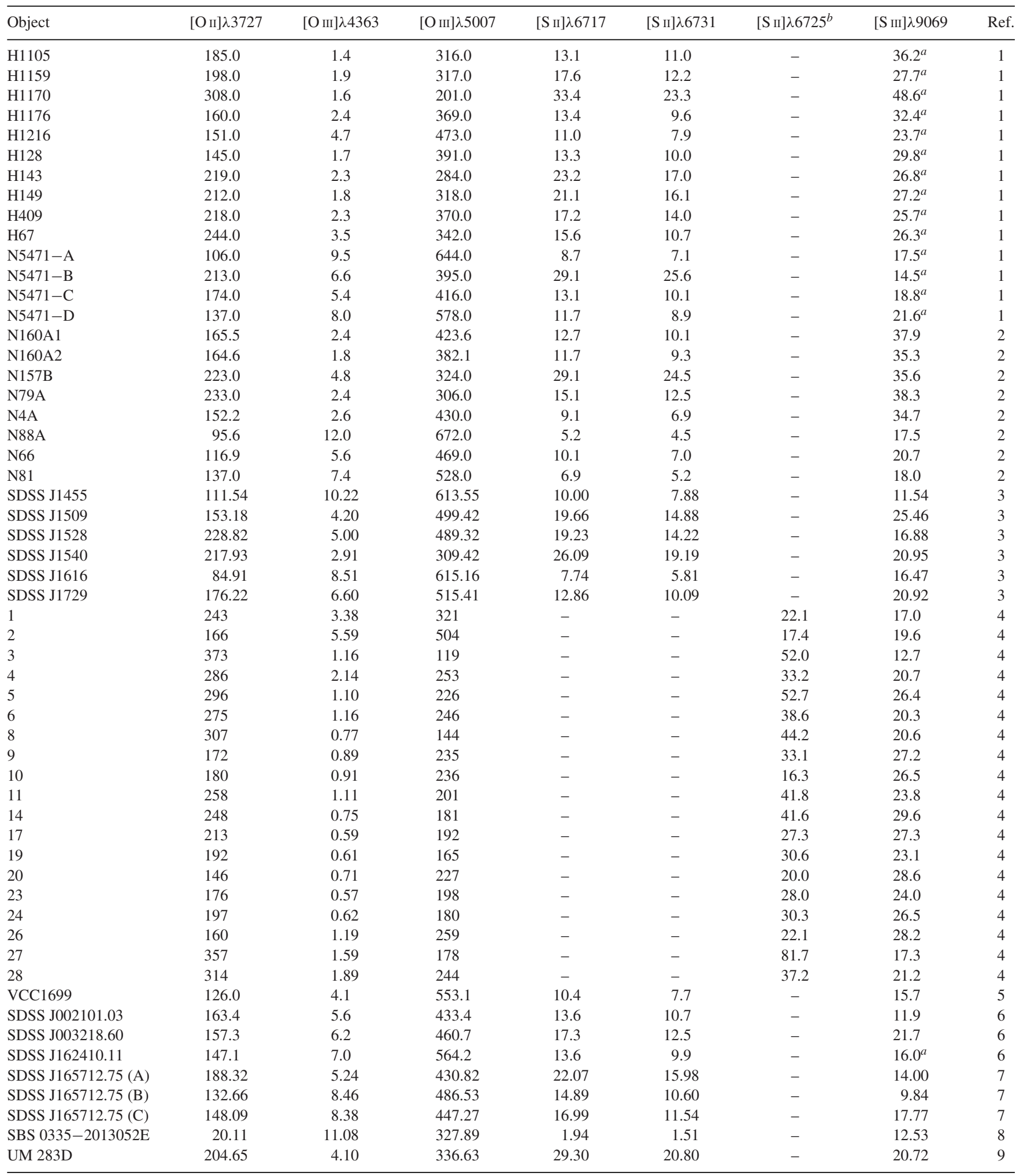


Table A1. - continued

\begin{tabular}{|c|c|c|c|c|c|c|c|c|}
\hline Object & {$\left[\mathrm{O}_{\text {II }}\right] \lambda 3727$} & {$[\mathrm{O}$ ІІा] $] \lambda 4363$} & {$[\mathrm{O}$ ІІІ $] \lambda 5007$} & {$\left[\mathrm{~S}_{\text {II }}\right] \lambda 6717$} & {$\left[\mathrm{~S}_{\text {II }}\right] \lambda 6731$} & {$\left[\mathrm{~S}_{\text {II }}\right] \lambda 6725^{b}$} & {$\left[\mathrm{~S}_{\text {III }}\right] \lambda 9069$} & Ref. \\
\hline HE $2-10 \mathrm{C}$ & 211.87 & 1.96 & 150.52 & 14.69 & 17.87 & - & 34.18 & 9 \\
\hline NGC 3125 & 93.12 & 5.13 & 578.95 & 11.97 & 9.92 & - & 31.97 & 9 \\
\hline Mrk 1259 & 170.87 & 1.08 & 161.78 & 15.75 & 18.13 & - & 23.74 & 9 \\
\hline POX 4 & 81.26 & 9.12 & 652.36 & 7.63 & 5.94 & - & 12.31 & 9 \\
\hline NGC 5253 No.C1 & 96.34 & 7.45 & 634.27 & 7.79 & 7.79 & - & 22.44 & 9 \\
\hline NGC 5253 No.C2 & 148.48 & 3.09 & 445.98 & 14.80 & 12.20 & - & 21.57 & 9 \\
\hline NGC 5253 No.P2 & 106.83 & 7.92 & 649.56 & 7.19 & 8.00 & - & 22.82 & 9 \\
\hline TOL 89 No. 1 & 151.60 & 3.38 & 489.58 & 11.67 & 8.99 & - & 24.83 & 9 \\
\hline TOL 89 No.2 & 157.62 & 1.94 & 385.19 & 24.69 & 18.48 & - & 50.12 & 9 \\
\hline TOL 1457-262 & 258.97 & 6.80 & 627.73 & 11.99 & 9.03 & - & 13.90 & 9 \\
\hline VS 49 & 219.0 & 2.9 & 239.0 & 23.8 & 16.9 & - & 25.5 & 10 \\
\hline VS 48 & 252.0 & 3.8 & 314.0 & 20.9 & 15.9 & - & 26.8 & 10 \\
\hline NGC 604 & 215.2 & 0.75 & 207.7 & 16.6 & 11.1 & - & 3.98 & 11 \\
\hline NGC 588 & 148.2 & 2.4 & 464.7 & 11.8 & 7.7 & - & 5.63 & 11 \\
\hline Leo P & 46.5 & 3.8 & 145.3 & 3.6 & 2.7 & - & 14.5 & 12 \\
\hline IC $132-\mathrm{A}$ & 150.7 & 6.7 & 500.1 & 8.9 & 5.7 & - & 27.5 & 13 \\
\hline IC $132-B$ & 164.7 & 6.4 & 485.6 & 9.9 & 6.6 & - & 24.0 & 13 \\
\hline IC $132-\mathrm{C}$ & 192.4 & 7.7 & 463.5 & 11.7 & 7.6 & - & 28.4 & 13 \\
\hline IC $132-\mathrm{D}$ & 210.5 & 8.4 & 449.1 & 12.7 & 8.4 & - & 30.8 & 13 \\
\hline IC $132-E$ & 204.5 & 10.7 & 443.5 & 12.3 & 8.2 & - & 30.6 & 13 \\
\hline IC $132-\mathrm{F}$ & 188.9 & 11.2 & 445.1 & 11.5 & 7.3 & - & 28.1 & 13 \\
\hline IC $132-\mathrm{G}$ & 221.6 & 14.0 & 435.0 & 12.9 & 8.6 & - & 28.2 & 13 \\
\hline IC $132-\mathrm{H}$ & 241.3 & 18.9 & 433.3 & 14.0 & 9.2 & - & 23.4 & 13 \\
\hline Mrk 5 & 212.9 & 4.4 & 381.5 & 23.3 & 16.6 & - & 15.9 & 15 \\
\hline $0749+568$ & 166.8 & 9.8 & 488.0 & 17.8 & 11.4 & - & 12.7 & 15 \\
\hline $0926+606$ & 178.5 & 8.3 & 477.2 & 18.2 & 14.6 & - & 12.2 & 15 \\
\hline Mrk 709 & 183.6 & 8.8 & 369.6 & 31.3 & 28.5 & - & 8.7 & 15 \\
\hline Mrk 22 & 148.7 & 8.2 & 545.5 & 11.4 & 8.5 & - & 14.8 & 15 \\
\hline Mrk 1434 & 96.8 & 10.4 & 502.8 & 9.6 & 6.7 & - & 9.2 & 15 \\
\hline Mrk 36 & 129.3 & 9.6 & 483.4 & 11.7 & 8.4 & - & 11.9 & 15 \\
\hline VII Zw 403 & 133.3 & 7.1 & 345.5 & 10.3 & 7.5 & - & 11.7 & 15 \\
\hline UM 461 & 52.7 & 13.6 & 602.2 & 5.2 & 4.2 & - & 12.4 & 15 \\
\hline UM 462 & 174.2 & 7.8 & 492.9 & 16.8 & 11.2 & - & 10.5 & 15 \\
\hline Mrk 209 & 71.9 & 12.7 & 554.3 & 6.1 & 4.5 & - & 12.2 & 15 \\
\hline A & 245 & 1.7 & 134.1 & 41.9 & 32.5 & - & 20.8 & 16 \\
\hline N110 & 182 & 1.3 & 140 & 29 & 28 & - & 27.0 & 16 \\
\hline B & 242 & 3.1 & 357 & 30.6 & 22.4 & - & 14.2 & 16 \\
\hline $\mathrm{C}$ & 280 & 1.7 & 257 & 33 & 24 & - & 13.0 & 16 \\
\hline $\mathrm{N}$ & 173 & 1.2 & 152 & 30 & 29 & - & 31.0 & 16 \\
\hline SE & 46.6 & 4.4 & 189 & - & - & 7.2 & 3.9 & 17 \\
\hline NW & 26.4 & 6.2 & 185 & - & - & 3.8 & 3.0 & 17 \\
\hline N12A & 191 & 3.7 & 368.4 & 9.2 & 7.4 & - & 19.9 & 18 \\
\hline N13AB & 216.3 & 2.7 & 347.6 & 13.4 & 8.4 & - & 23.6 & 18 \\
\hline $\mathrm{N} 4 \mathrm{~A}$ & 203.5 & 2.6 & 394.4 & 13.4 & 11.0 & - & 36.7 & 18 \\
\hline N138A & 179.1 & 2.1 & 371.0 & 8.0 & 6.2 & - & 28.0 & 18 \\
\hline Haro $15-B$ & 90.5 & 7.8 & 698 & 7.3 & 5.9 & - & 11.9 & 19 \\
\hline
\end{tabular}


Table A2. Fluxes of infrared emission lines (in $\mathrm{W} / \mathrm{cm}^{2}$ ) compiled from the literature. References - (19) data compiled from Peeters et al. (2002), (20) Vermeij et al. (2002), (21) Lebouteiller et al. (2008), (22) Rubin et al. (2008), (23) Simpson et al. (1998), (24) Simpson et al. (2012), (25) Bernard-Salas et al. (2009), (26) Giveon et al. (2002), and (27) Nollenberg et al. (2002).

\begin{tabular}{|c|c|c|c|c|c|}
\hline Object & H I $4.05 \mu \mathrm{m}$ & {$\left[\mathrm{S}_{\text {IV }}\right] 10.51 \mu \mathrm{m}$} & {$\left[\mathrm{S}_{\mathrm{III}}\right] 18.71 \mu \mathrm{m}$} & Flux W/ $\mathrm{cm}^{2}$ & Reference \\
\hline IR 02219 & 6.6 & 26 & 59 & $10^{-18}$ & 19 \\
\hline IR 02219 & 6.7 & 28 & 43 & $10^{-18}$ & 19 \\
\hline IR 10589 & 1.71 & 1.5 & 15.7 & $10^{-18}$ & 19 \\
\hline IR 11143 & 0.68 & 8.4 & 7.8 & $10^{-18}$ & 19 \\
\hline IR 12063 & 2.08 & 9.1 & 11.8 & $10^{-18}$ & 19 \\
\hline IR 12073 & 6.6 & 59 & 35 & $10^{-18}$ & 19 \\
\hline IR 12331 & 0.51 & 1.28 & 9.3 & $10^{-18}$ & 19 \\
\hline IR 15384 & 2.86 & 2.6 & 25.9 & $10^{-18}$ & 19 \\
\hline IR 15502 & 1.5 & 0.23 & 4.9 & $10^{-18}$ & 19 \\
\hline IR 16128 & 0.63 & 0.82 & 6.4 & $10^{-18}$ & 19 \\
\hline IR 17221 & 0.64 & 0.13 & 7.3 & $10^{-18}$ & 19 \\
\hline IR 17455 & 2.0 & 1.9 & 23.2 & $10^{-18}$ & 19 \\
\hline IR 18317 & 2.00 & 0.33 & 25.6 & $10^{-18}$ & 19 \\
\hline IR 18434 & 6.80 & 4.2 & 46 & $10^{-18}$ & 19 \\
\hline IR 18502 & 0.89 & 0.49 & 8.6 & $10^{-18}$ & 19 \\
\hline IR 19207 & 0.67 & 1.41 & 7.4 & $10^{-18}$ & 19 \\
\hline IR 19598 & 4.7 & 3.3 & 7.6 & $10^{-18}$ & 19 \\
\hline IR 21190 & 2.25 & 2.09 & 6.51 & $10^{-18}$ & 19 \\
\hline IR 23030 & 1.60 & 1.3 & 16.3 & $10^{-18}$ & 19 \\
\hline IR 23133 & 1.74 & 0.20 & 10.0 & $10^{-18}$ & 19 \\
\hline N160A1 & 51.7 & 289 & 317 & $10^{-20}$ & 20 \\
\hline N160A2 & 55.2 & 194 & 268 & $10^{-20}$ & 20 \\
\hline N159-5 & 12.6 & 56.7 & 74.2 & $10^{-20}$ & 20 \\
\hline $\mathrm{N} 4 \mathrm{~A}$ & 10.7 & 75.9 & 93.6 & $10^{-20}$ & 20 \\
\hline N83B & 12.3 & 18.1 & 28.8 & $10^{-20}$ & 20 \\
\hline N157B & $6.3^{a}$ & 11.7 & 46.5 & $10^{-20}$ & 20 \\
\hline N88A & $14.5^{a}$ & 55.1 & 8.89 & $10^{-20}$ & 20 \\
\hline N81 & $9.7^{a}$ & 15.9 & 14.1 & $10^{-20}$ & 20 \\
\hline NGC $3603^{c}$ & $101.07^{b}$ & 186.94 & 691.99 & $10^{-20}$ & 21 \\
\hline 30 Dor $^{c}$ & $168.02^{b}$ & 811.68 & 802.4 & $10^{-20}$ & 21 \\
\hline $\mathrm{N}^{6} 6^{c}$ & $8.754^{b}$ & 25.72 & 23.44 & $10^{-20}$ & 21 \\
\hline 638 & $1.37^{b}$ & 4.94 & 3.09 & $10^{-20}$ & 22 \\
\hline 623 & $2.98^{b}$ & 7.45 & 5.04 & $10^{-20}$ & 22 \\
\hline 45 & $3.55^{b}$ & 10.1 & 17.4 & $10^{-20}$ & 22 \\
\hline 214 & $1.39^{b}$ & 54.2 & 5.18 & $10^{-20}$ & 22 \\
\hline 33 & $0.901^{b}$ & 1.22 & 4.91 & $10^{-20}$ & 22 \\
\hline 42 & $0.625^{b}$ & 0.573 & 2.56 & $10^{-20}$ & 22 \\
\hline 32 & $0.657^{b}$ & 0.238 & 1.48 & $10^{-20}$ & 22 \\
\hline 251 & $1.00^{b}$ & 1.57 & 3.02 & $10^{-20}$ & 22 \\
\hline 301 & $0.915^{b}$ & 0.475 & 4.69 & $10^{-20}$ & 22 \\
\hline 4 & $1.78^{b}$ & 1.46 & 7.80 & $10^{-20}$ & 22 \\
\hline 79 & $3.00^{b}$ & 7.70 & 23.1 & $10^{-20}$ & 22 \\
\hline $87 \mathrm{E}$ & $9.30^{b}$ & 5.29 & 49.1 & $10^{-21}$ & 22 \\
\hline 302 & $9.65^{b}$ & 7.26 & 39.8 & $10^{-21}$ & 22 \\
\hline 95 & $6.10^{b}$ & 6.95 & 36.8 & $10^{-21}$ & 22 \\
\hline 710 & $1.51^{b}$ & 0.659 & 4.05 & $10^{-20}$ & 22 \\
\hline 691 & $1.18^{b}$ & 4.43 & 6.43 & $10^{-20}$ & 22 \\
\hline Orion & $1.448^{b}$ & 4.765 & 14.49 & $10^{-10}$ & 23 \\
\hline G333-North & $2.932^{b}$ & 0.256 & 14.52 & $10^{-11}$ & 24 \\
\hline G333-West & $2.93^{b}$ & 0.164 & 17.51 & $10^{-11}$ & 24 \\
\hline NGC 1222 & $15.05^{b}$ & 22.24 & 64.80 & $10^{-21}$ & 25 \\
\hline IC 342 & $44.16^{b}$ & 4.76 & 320.03 & $10^{-21}$ & 25 \\
\hline NGC 1614 & $12.56^{b}$ & 6.89 & 83.03 & $10^{-21}$ & 25 \\
\hline NGC 2146 & $48.35^{b}$ & 6.30 & 190.12 & $10^{-21}$ & 25 \\
\hline NGC 3256 & $43.94^{b}$ & 5.25 & 171.83 & $10^{-21}$ & 25 \\
\hline NGC 3310 & $4.11^{b}$ & 4.46 & 20.50 & $10^{-21}$ & 25 \\
\hline NGC 4676 & $3.40^{b}$ & 0.56 & 11.32 & $10^{-21}$ & 25 \\
\hline NGC 4818 & $12.14^{b}$ & 2.21 & 71.96 & $10^{-21}$ & 25 \\
\hline NGC 7714 & $15.47^{b}$ & 14.76 & 81.50 & $10^{-21}$ & 25 \\
\hline
\end{tabular}


Table A2. - continued

\begin{tabular}{|c|c|c|c|c|c|}
\hline Object & H I $4.05 \mu \mathrm{m}$ & {$[\mathrm{S}$ IV $] 10.51 \mu \mathrm{m}$} & {$\left[\mathrm{S}_{\mathrm{III}}\right] 18.71 \mu \mathrm{m}$} & Flux $\mathrm{W} / \mathrm{cm}^{2}$ & Reference \\
\hline W3 IRS 5 & 3.5 & 3.3 & 16.6 & $10^{-19}$ & 26 \\
\hline W3 IRS 2 & 63.3 & 228 & 570 & $10^{-19}$ & 26 \\
\hline W3 IRS 2 & 63.5 & 259 & 414 & $10^{-19}$ & 26 \\
\hline $\mathrm{ORION}^{c}$ & 373.9 & 1116.7 & 3659.0 & $10^{-19}$ & 26 \\
\hline Caswell H2O 287.37-00.62 & 1.6 & 2.8 & 29.8 & $10^{-19}$ & 26 \\
\hline TRUMPLER14 & 6.4 & 9.6 & 68.7 & $10^{-19}$ & 26 \\
\hline Gal 287.39-00.63 & 5.6 & 34.0 & 63.7 & $10^{-19}$ & 26 \\
\hline Gal $289.88-00.79$ & 16.3 & 14.6 & 149.0 & $10^{-19}$ & 26 \\
\hline NGC 3603 & 1.7 & 15.7 & 25.4 & $10^{-19}$ & 26 \\
\hline RAFGL 4127 & 6.6 & 75.7 & 72.9 & $10^{-19}$ & 26 \\
\hline Gal 298.23-00.33 & 65.9 & 557.0 & 382.0 & $10^{-19}$ & 26 \\
\hline GRS $301.11+00.97$ & 4.7 & 11.5 & 77.3 & $10^{-19}$ & 26 \\
\hline GRS $326.44+00.91$ & 26.4 & 25.5 & 254.0 & $10^{-19}$ & 26 \\
\hline $15408-5356$ & 29.7 & 53.4 & 441.0 & $10^{-19}$ & 26 \\
\hline G327.3-0.5 & 28.7 & 27.5 & 497.0 & $10^{-19}$ & 26 \\
\hline GRS $328.30+00.43$ & 14.7 & 2.4 & 48.8 & $10^{-19}$ & 26 \\
\hline $15567-5236$ & 25.7 & 6.4 & 40.4 & $10^{-19}$ & 26 \\
\hline $16172-5028$ & 12.7 & 4.8 & 136.0 & $10^{-19}$ & 26 \\
\hline G333.13-0.43 & 4.4 & 12.0 & 100.0 & $10^{-19}$ & 26 \\
\hline Gal 337.9-00.5 & 15.2 & 30.0 & 255.0 & $10^{-19}$ & 26 \\
\hline $17059-4132$ & 17.7 & 6.7 & 398.0 & $10^{-19}$ & 26 \\
\hline NGC 6334-A & 5.0 & 6.8 & 44.3 & $10^{-19}$ & 26 \\
\hline NGC 637I & 11.5 & 51.0 & 251.0 & $10^{-19}$ & 26 \\
\hline Gal 351.47-00.46 & 5.5 & 1.5 & 63.1 & $10^{-19}$ & 26 \\
\hline NGC 6357IIIB & 4.0 & 9.6 & 86.4 & $10^{-19}$ & 26 \\
\hline RAFGL 2003 & 46.6 & 5.8 & 149.0 & $10^{-19}$ & 26 \\
\hline ARCHFIL $^{c}$ & 13.91 & 0.9 & 133.2 & $10^{-19}$ & 26 \\
\hline Pistol star & 7.1 & 1.3 & 32.2 & $10^{-19}$ & 26 \\
\hline SGR D H II & 18.8 & 16.8 & 201.0 & $10^{-19}$ & 26 \\
\hline RAFGL 2094 & 11.1 & 1.7 & 138.0 & $10^{-19}$ & 26 \\
\hline $\mathrm{M} 17^{c}$ & 184.23 & 1087.1 & 2457.2 & $10^{-19}$ & 26 \\
\hline $18317-0757$ & 18.8 & 2.9 & 260.0 & $10^{-19}$ & 26 \\
\hline RAFGL 2245 & 13.9 & 39.0 & 357.0 & $10^{-19}$ & 26 \\
\hline Gal $033.91+00.11$ & 8.8 & 59.9 & 5.1 & $10^{-19}$ & 26 \\
\hline Gal $045.45+00.06$ & 12.0 & 19.8 & 119.0 & $10^{-19}$ & 26 \\
\hline Gal $049.20-00.35$ & 6.4 & 12.1 & 76.6 & $10^{-19}$ & 26 \\
\hline W51 IRS2 & 77.4 & 137.0 & 337.0 & $10^{-19}$ & 26 \\
\hline IR $070.29+0160^{c}$ & 89.8 & 57.6 & 153.0 & $10^{-19}$ & 26 \\
\hline S128 A & 2.5 & 4.0 & 21.0 & $10^{-19}$ & 26 \\
\hline S138 & 6.0 & 1.1 & 44.0 & $10^{-19}$ & 26 \\
\hline S156 A & 15.5 & 11.8 & 170.0 & $10^{-19}$ & 26 \\
\hline S159 & 16.4 & 1.9 & 101.0 & $10^{-19}$ & 26 \\
\hline Hubble V & 1.9 & 7.5 & 6.7 & $10^{-20}$ & 27 \\
\hline I Zw 36 & 1.9 & 12 & 4.9 & $10^{-21}$ & 27 \\
\hline
\end{tabular}

Notes. ${ }^{a}$ Flux computed from $\mathrm{H}_{\mathrm{I}} 4.051 \mu \mathrm{m} / \mathrm{H}_{\mathrm{I}} 2.63 \mu \mathrm{m}=1.74$.

${ }^{b}$ Flux computed from $\mathrm{H}_{\mathrm{I}} 4.051 \mu \mathrm{m} / \mathrm{H}_{\text {I }} 12.37 \mu \mathrm{m}=8.2$.

${ }^{c}$ Flux obtained assuming the measurements at the different observed positions. 
Table A3. Electron temperature, electron density, ionic and total oxygen abundances for the sample of objects listed in Table A1. The estimations were calculated following the Visible-lines method described in Section 4.1. For the cases where was not possible to calculate the electron density, a value of $N_{\mathrm{e}}=200 \mathrm{~cm}^{-3}$ was assumed.

\begin{tabular}{|c|c|c|c|c|c|c|}
\hline Object & $T_{\mathrm{e}}\left(10^{4}\right) \mathrm{K}$ & $N_{\mathrm{e}}\left(\mathrm{cm}^{-3}\right)$ & $\log \left(\mathrm{O}^{+} / \mathrm{H}^{+}\right)$ & $12+\log (\mathrm{O} / \mathrm{H})$ & $\log \left(\mathrm{S}^{+} / \mathrm{H}^{+}\right)$ & $\log \left(\mathrm{S}^{+2} / \mathrm{H}^{+}\right)$ \\
\hline H1105 & 0.9046 & 211 & 7.92 & 8.39 & 5.85 & 6.87 \\
\hline H1159 & 0.9721 & - & 7.84 & 8.29 & 5.89 & 6.68 \\
\hline H1170 & 1.0512 & - & 7.93 & 8.16 & 6.10 & 6.85 \\
\hline H1176 & 0.9931 & 37 & 7.72 & 8.27 & 5.76 & 6.73 \\
\hline H1216 & 1.1277 & 37 & 7.53 & 8.17 & 5.58 & 6.47 \\
\hline H128 & 0.9008 & 95 & 7.82 & 8.43 & 5.84 & 6.79 \\
\hline H143 & 1.0566 & 58 & 7.77 & 8.16 & 5.95 & 6.59 \\
\hline H149 & 0.9582 & 101 & 7.89 & 8.32 & 5.99 & 6.69 \\
\hline H409 & 0.9813 & 176 & 7.87 & 8.33 & 5.90 & 6.64 \\
\hline H67 & 1.1390 & - & 7.73 & 8.13 & 5.71 & 6.51 \\
\hline N5471-A & 1.3090 & 188 & 7.21 & 8.07 & 5.40 & 6.22 \\
\hline N5471-B & 1.3817 & 301 & 7.46 & 7.91 & 5.91 & 6.09 \\
\hline N5471-C & 1.2428 & 115 & 7.48 & 8.02 & 5.60 & 6.29 \\
\hline N5471-D & 1.2750 & 99 & 7.35 & 8.08 & 5.53 & 6.33 \\
\hline N160A1 & 0.9584 & 147 & 7.78 & 8.38 & 5.78 & 6.81 \\
\hline N160A2 & 0.9172 & 145 & 7.84 & 8.41 & 5.78 & 6.82 \\
\hline N157B & 1.3113 & 232 & 7.53 & 7.93 & 5.93 & 6.51 \\
\hline N79A & 1.0466 & 200 & 7.81 & 8.20 & 5.80 & 6.73 \\
\hline N4A & 0.9743 & 94 & 7.72 & 8.35 & 5.61 & 6.74 \\
\hline N88A & 1.4242 & 228 & 7.08 & 7.98 & 5.14 & 5.95 \\
\hline N66 & 1.2037 & - & 7.35 & 8.06 & 5.49 & 6.32 \\
\hline N81 & 1.2815 & 88 & 7.34 & 8.04 & 5.30 & 6.19 \\
\hline SDSS J1455 & 1.3798 & 142 & 7.18 & 7.99 & 5.42 & 6.04 \\
\hline SDSS J1509 & 1.0688 & 93 & 7.61 & 8.26 & 5.88 & 6.49 \\
\hline SDSS J1528 & 1.1384 & 67 & 7.70 & 8.22 & 5.82 & 6.30 \\
\hline SDSS J1540 & 1.1073 & 62 & 7.71 & 8.12 & 5.97 & 6.44 \\
\hline SDSS J1616 & 1.2747 & 83 & 7.14 & 8.07 & 5.35 & 6.20 \\
\hline SDSS J1729 & 1.2362 & 136 & 7.49 & 8.10 & 5.60 & 6.31 \\
\hline 1 & 1.1503 & - & 7.72 & 8.10 & 5.63 & 6.31 \\
\hline 2 & 1.1716 & - & 7.53 & 8.15 & 5.51 & 6.36 \\
\hline 3 & 1.1205 & - & 7.93 & 8.06 & 6.02 & 6.21 \\
\hline 4 & 1.0707 & - & 7.87 & 8.17 & 5.86 & 6.46 \\
\hline 5 & 0.9241 & - & 8.09 & 8.36 & 6.18 & 6.71 \\
\hline 6 & 0.9174 & - & 8.07 & 8.37 & 6.05 & 6.61 \\
\hline 8 & 0.9449 & - & 8.07 & 8.26 & 6.08 & 6.58 \\
\hline 9 & 0.8742 & - & 7.93 & 8.35 & 6.02 & 6.79 \\
\hline 10 & 0.8776 & - & 7.95 & 8.35 & 5.71 & 6.77 \\
\hline 11 & 0.9524 & - & 7.99 & 8.26 & 6.05 & 6.64 \\
\hline 14 & 0.8913 & - & 8.06 & 8.33 & 6.10 & 6.80 \\
\hline 17 & 0.8371 & - & 8.09 & 8.41 & 5.98 & 6.84 \\
\hline 19 & 0.8698 & - & 7.99 & 8.29 & 5.99 & 6.72 \\
\hline 20 & 0.8402 & - & 7.93 & 8.38 & 5.84 & 6.85 \\
\hline 23 & 0.8261 & - & 8.03 & 8.40 & 6.00 & 6.80 \\
\hline 24 & 0.8570 & - & 8.02 & 8.34 & 6.00 & 6.80 \\
\hline 26 & 0.9120 & - & 7.84 & 8.30 & 5.81 & 6.76 \\
\hline 27 & 1.0891 & - & 7.95 & 8.14 & 6.24 & 6.37 \\
\hline 28 & 1.0428 & - & 7.95 & 8.22 & 5.93 & 6.50 \\
\hline VCC1699 & 1.0296 & 69 & 7.57 & 8.34 & 5.62 & 6.49 \\
\hline SDSS J002101.03 & 1.2406 & 139 & 7.46 & 8.03 & 5.62 & 6.03 \\
\hline SDSS J003218.60 & 1.2607 & 41 & 7.42 & 8.03 & 5.70 & 6.28 \\
\hline SDSS J162410.11 & 1.2213 & 50 & 7.43 & 8.13 & 5.62 & 6.16 \\
\hline SDSS J165712.75 (A) & 1.2121 & 44 & 7.54 & 8.07 & 5.83 & 6.20 \\
\hline SDSS J165712.75 (B) & 1.4069 & 24 & 7.23 & 7.90 & 5.56 & 5.94 \\
\hline SDSS J165712.75 (C) & 1.4567 & - & 7.25 & 7.85 & 5.59 & 6.00 \\
\hline SBS 0335-2013052E & 2.0093 & 126 & 6.11 & 7.30 & 4.52 & 5.24 \\
\hline UM 283D & 1.2127 & 24 & 7.58 & 8.01 & 5.95 & 6.35 \\
\hline UM 133H & 1.5396 & 63 & 7.06 & 7.69 & 5.32 & 5.72 \\
\hline HE $2-10 \mathrm{C}$ & 1.2443 & 1082 & 7.57 & 7.81 & 5.75 & 6.55 \\
\hline HE $2-10 \mathrm{E}$ & 0.7708 & 723 & 8.19 & 8.44 & 6.10 & 6.94 \\
\hline
\end{tabular}


Table A3. - continued

\begin{tabular}{|c|c|c|c|c|c|c|}
\hline Object & $T_{\mathrm{e}}\left(10^{4}\right) \mathrm{K}$ & $N_{\mathrm{e}}\left(\mathrm{cm}^{-3}\right)$ & $\log \left(\mathrm{O}^{+} / \mathrm{H}^{+}\right)$ & $12+\log (\mathrm{O} / \mathrm{H})$ & $\log \left(\mathrm{S}^{+} / \mathrm{H}^{+}\right)$ & $\log \left(\mathrm{S}^{+2} / \mathrm{H}^{+}\right)$ \\
\hline NGC 3125 & 1.0863 & 203 & 7.37 & 8.26 & 5.67 & 6.64 \\
\hline Mrk 1259 & 1.0001 & 841 & 7.74 & 8.05 & 5.92 & 6.59 \\
\hline POX 4 & 1.2802 & 127 & 7.12 & 8.08 & 5.35 & 6.08 \\
\hline TOL 1214-277 & 2.0090 & - & 6.30 & 7.49 & 4.54 & 5.14 \\
\hline J $1253-0312$ & 1.3398 & 1257 & 7.09 & 8.04 & 5.24 & 6.09 \\
\hline NGC 5253 No.C1 & 1.1964 & 526 & 7.27 & 8.17 & 5.45 & 6.40 \\
\hline NGC 5253 No.C2 & 1.0102 & 193 & 7.66 & 8.30 & 5.81 & 6.53 \\
\hline NGC 5253 No.P & 1.2132 & 789 & 7.30 & 8.16 & 5.43 & 6.39 \\
\hline TOL 89 No. 1 & 1.0093 & 112 & 7.68 & 8.34 & 5.70 & 6.60 \\
\hline TOL 89 No. 2 & 0.9315 & 80 & 7.80 & 8.38 & 6.08 & 6.98 \\
\hline TOL 1457-262 & 1.1618 & 87 & 7.73 & 8.28 & 5.60 & 6.21 \\
\hline TOL $1924-426$ No.1 & 1.2169 & 105 & 7.24 & 8.00 & 5.47 & 6.15 \\
\hline TOL $1924-426$ No.2 & 1.1954 & 143 & 7.34 & 8.11 & 5.55 & 6.19 \\
\hline NGC $6822 \mathrm{~V}$ & 1.1212 & 107 & 7.26 & 8.17 & 5.33 & 6.51 \\
\hline VS 3 & 1.0052 & 28 & 7.85 & 8.09 & 5.90 & 6.55 \\
\hline VS 49 & 1.2110 & 31 & 7.61 & 7.94 & 5.86 & 6.36 \\
\hline VS 48 & 1.2098 & 99 & 7.67 & 8.03 & 5.82 & 6.41 \\
\hline NGC 604 & 0.8655 & - & 8.05 & 8.38 & 5.95 & 5.76 \\
\hline NGC 588 & 0.9371 & - & 7.77 & 8.43 & 5.73 & 6.04 \\
\hline Leo P & 1.7296 & 80 & 6.59 & 7.18 & 4.85 & 5.39 \\
\hline IC $132-\mathrm{A}$ & 1.2584 & - & 7.41 & 8.05 & 5.39 & 6.44 \\
\hline IC $132-B$ & 1.2503 & - & 7.45 & 8.06 & 5.45 & 6.39 \\
\hline IC $132-\mathrm{C}$ & 1.3781 & - & 7.42 & 7.95 & 5.45 & 6.39 \\
\hline IC $132-D$ & 1.4555 & - & 7.40 & 7.89 & 5.46 & 6.38 \\
\hline IC $132-\mathrm{E}$ & 1.6552 & - & 7.27 & 7.75 & 5.38 & 6.29 \\
\hline IC $132-\mathrm{F}$ & 1.6932 & - & 7.22 & 7.72 & 5.33 & 6.24 \\
\hline IC $132-\mathrm{G}$ & 1.9505 & - & 7.18 & 7.61 & 5.32 & 6.15 \\
\hline IC $132-\mathrm{H}$ & 2.3685 & - & 7.08 & 7.48 & 5.28 & 5.96 \\
\hline IC $132-I$ & 3.1568 & - & 6.97 & 7.32 & 5.23 & 5.73 \\
\hline 8 & 1.2283 & 190 & 7.43 & 7.51 & 6.14 & 6.30 \\
\hline 17 & 0.8603 & 60 & 7.88 & 8.17 & 5.93 & 6.73 \\
\hline 25 & 0.9377 & 48 & 7.79 & 8.24 & 5.72 & 6.60 \\
\hline 26 & 0.9495 & 16 & 7.94 & 8.26 & 5.91 & 6.61 \\
\hline 35 & 0.8948 & 48 & 7.84 & 8.38 & 5.82 & 6.65 \\
\hline 32 & 1.1113 & 73 & 7.80 & 7.95 & 6.14 & 6.32 \\
\hline II Zw 40 & 1.3075 & 171 & 7.11 & 8.11 & 5.28 & 6.05 \\
\hline Mrk 5 & 1.1884 & 28 & 7.62 & 8.08 & 5.86 & 6.19 \\
\hline $0749+568$ & 1.5064 & - & 7.27 & 7.85 & 5.58 & 5.97 \\
\hline $0926+606$ & 1.4070 & 166 & 7.36 & 7.93 & 5.67 & 6.06 \\
\hline Mrk 709 & 1.6435 & 366 & 7.23 & 7.69 & 5.85 & 5.75 \\
\hline Mrk 22 & 1.3194 & 75 & 7.35 & 8.02 & 5.49 & 6.14 \\
\hline Mrk 1434 & 1.5286 & - & 7.02 & 7.80 & 5.32 & 5.82 \\
\hline Mrk 36 & 1.4982 & 32 & 7.16 & 7.83 & 5.42 & 5.95 \\
\hline VII Zw 403 & 1.5236 & 47 & 7.16 & 7.70 & 5.36 & 5.93 \\
\hline UM 461 & 1.5985 & 176 & 6.71 & 7.79 & 5.06 & 5.82 \\
\hline UM 462 & 1.3489 & - & 7.40 & 7.98 & 5.63 & 6.06 \\
\hline Mrk 209 & 1.6105 & 61 & 6.84 & 7.76 & 5.11 & 5.91 \\
\hline A & 1.2314 & 122 & 7.64 & 7.84 & 6.11 & 6.38 \\
\hline N110 & 1.1027 & 447 & 7.64 & 7.90 & 6.07 & 6.56 \\
\hline B & 1.0794 & 57 & 7.79 & 8.21 & 6.06 & 6.29 \\
\hline $\mathrm{C}$ & 0.9976 & 51 & 7.96 & 8.26 & 6.15 & 6.29 \\
\hline $\mathrm{N}$ & 1.0486 & 443 & 7.68 & 7.98 & 6.12 & 6.65 \\
\hline SE & 1.6241 & - & 6.65 & 7.33 & 4.94 & 5.25 \\
\hline NW & 1.9992 & - . & 6.24 & 7.09 & 4.56 & 5.06 \\
\hline N12A & 1.1318 & 165 & 7.63 & 8.12 & 5.52 & 6.39 \\
\hline $\mathrm{N} 13 \mathrm{AB}$ & 1.0436 & - & 7.79 & 8.23 & 5.69 & 6.54 \\
\hline $\mathrm{N} 4 \mathrm{~A}$ & 0.9967 & 187 & 7.82 & 8.32 & 5.78 & 6.78 \\
\hline N138A & 0.9582 & 118 & 7.82 & 8.34 & 5.58 & 6.70 \\
\hline Haro $15-\mathrm{B} 1.1748$ & 173 & 7.26 & 8.22 & 5.39 & 5.59 & \\
\hline
\end{tabular}


Table A4. Ionic abundances for the sample of objects obtained listed in Table A2 calculated following the IR-lines method described in Section 4.2 and assuming an electron temperature of $10000 \mathrm{~K}$.

\begin{tabular}{|c|c|c|c|c|c|}
\hline Object & $12+\log \left(\mathrm{S}^{2+} / \mathrm{H}^{+}\right)$ & $12+\log \left(\mathrm{S}^{3+} / \mathrm{H}^{+}\right)$ & Object & $12+\log \left(\mathrm{S}^{2+} / \mathrm{H}^{+}\right)$ & $12+\log \left(\mathrm{S}^{3+} / \mathrm{H}^{+}\right)$ \\
\hline IR 02219 & 6.83 & 5.80 & W3 IRS 5 & 6.56 & 5.18 \\
\hline IR 02219 & 6.69 & 5.82 & W3 IRS 2 & 6.84 & 5.76 \\
\hline IR 10589 & 6.85 & 5.14 & W3 IRS 2 & 6.70 & 5.81 \\
\hline IR 11143 & 6.94 & 6.29 & ORION & 6.87 & 5.68 \\
\hline IR 12063 & 6.64 & 5.84 & Caswell H2O 287.37-00.62 & 7.15 & 5.44 \\
\hline IR 12073 & 6.61 & 6.15 & TRUMPLER14 & 6.91 & 5.38 \\
\hline IR 12331 & 7.14 & 5.60 & Gal 287.39-00.63 & 6.94 & 5.98 \\
\hline IR 15384 & 6.84 & 5.16 & Gal 289.88-00.79 & 6.84 & 5.15 \\
\hline IR 15502 & 6.40 & 4.39 & NGC 3603 & 7.06 & 6.17 \\
\hline IR 16128 & 6.89 & 5.32 & RAFGL 4127 & 6.93 & 6.26 \\
\hline IR 17221 & 6.94 & 4.51 & Gal 298.23-00.33 & 6.65 & 6.13 \\
\hline IR 17455 & 6.95 & 5.18 & GRS $301.11+00.97$ & 7.10 & 5.59 \\
\hline IR 18317 & 6.99 & 4.42 & GRS 326.44+00.91 & 6.87 & 5.19 \\
\hline IR 18434 & 6.71 & 4.99 & $15408-5356$ & 7.05 & 5.46 \\
\hline IR 18502 & 6.87 & 4.94 & G327.3-0.5 & 7.12 & 5.18 \\
\hline IR 19207 & 6.93 & 5.52 & GRS $328.30+00.43$ & 6.40 & 4.41 \\
\hline IR 19598 & 6.09 & 5.05 & $15567-5236$ & 6.08 & 4.60 \\
\hline IR 21190 & 6.34 & 5.17 & $16172-5028$ & 6.91 & 4.78 \\
\hline IR 23030 & 6.89 & 5.11 & G333.13-0.43 & 7.24 & 5.64 \\
\hline IR 23133 & 6.64 & 4.26 & Gal 337.9-00.5 & 7.11 & 5.50 \\
\hline N160A1 & 6.67 & 5.95 & $17059-4132$ & 7.24 & 4.78 \\
\hline N160A2 & 6.57 & 5.75 & NGC 6334-A & 6.83 & 5.33 \\
\hline N159-5 & 6.65 & 5.85 & NGC 637I & 7.22 & 5.85 \\
\hline $\mathrm{N} 4 \mathrm{~A}$ & 6.83 & 6.05 & Gal 351.47-00.46 & 6.94 & 4.64 \\
\hline N83B & 6.25 & 5.37 & NGC 6357IIIB & 7.22 & 5.58 \\
\hline NGC 3603 & 6.72 & 5.47 & RAFGL 2003 & 6.39 & 4.30 \\
\hline 30 Dor & 6.56 & 5.89 & ARCHFIL & 6.86 & 4.01 \\
\hline 638 & 6.24 & 5.76 & Pistol star & 6.54 & 4.46 \\
\hline 623 & 6.11 & 5.60 & SGR D H II & 6.91 & 5.15 \\
\hline 45 & 6.57 & 5.66 & RAFGL 2094 & 6.98 & 4.39 \\
\hline 214 & 6.45 & 6.79 & M 17 & 7.01 & 5.97 \\
\hline 33 & 6.62 & 5.33 & $183170757 \mathrm{i}$ & 7.02 & 4.39 \\
\hline 42 & 6.50 & 5.16 & RAFGL 2245 & 7.29 & 5.65 \\
\hline 32 & 6.24 & 4.76 & Gal 033.91+00.11 & 5.65 & 6.03 \\
\hline 251 & 6.36 & 5.40 & Gal 045.45+00.06 & 6.88 & 5.42 \\
\hline 301 & 6.59 & 4.92 & Gal 049.20-00.35 & 6.96 & 5.48 \\
\hline 4 & 6.52 & 5.12 & W51 IRS2 & 6.52 & 5.45 \\
\hline 79 & 6.77 & 5.61 & S128 A & 6.81 & 5.41 \\
\hline $87 \mathrm{E}$ & 6.61 & 4.96 & $\mathrm{~S} 138$ & 6.75 & 4.46 \\
\hline 302 & 6.50 & 5.08 & S156 A & 6.92 & 5.08 \\
\hline 95 & 6.66 & 5.26 & S159 & 6.67 & 4.27 \\
\hline 710 & 6.31 & 4.84 & & & \\
\hline 691 & 6.62 & 5.78 & & & \\
\hline Orion & 6.88 & 5.72 & & & \\
\hline G333-North & 6.58 & 4.14 & & & \\
\hline G333-West & 6.66 & 3.95 & & & \\
\hline NGC 1222 & 6.52 & 5.37 & & & \\
\hline IC 342 & 6.74 & 4.23 & & & \\
\hline NGC 1614 & 6.70 & 4.94 & & & \\
\hline NGC 2146 & 6.48 & 4.32 & & & \\
\hline NGC 3256 & 6.48 & 4.28 & & & \\
\hline NGC 3310 & 6.58 & 5.24 & & & \\
\hline NGC 4676 & 6.41 & 4.42 & & & \\
\hline NGC 4818 & 6.66 & 4.46 & & & \\
\hline NGC 7714 & 6.60 & 5.18 & & & \\
\hline
\end{tabular}

This paper has been typeset from a $\mathrm{T}_{\mathrm{E}} \mathrm{X} / \mathrm{L} \mathrm{T} \mathrm{E} \mathrm{X}$ file prepared by the author. 